\title{
On regularity of a boundary point for higher-order parabolic equations: towards Petrovskii-type criterion by blow-up approach
}

\author{
Dedicated to the memory of Professor I.G. Petrovskii
}

\section{A. Galaktionov}

\begin{abstract}
The classic problem of regularity of boundary points for higherorder partial differential equations (PDEs) is concerned. For second-order elliptic and parabolic equations, this study was completed by Wiener's (J. Math. Phys. Mass. Inst. Tech. 3:127-146, 1924) and Petrovskii's (Math. Ann. 109:424-444, 1934) criteria, and was extended to more general equations including quasilinear ones. Since the 1960-1970s, the main success was achieved for $2 m$ th-order elliptic PDEs; e.g., by Kondrat'ev and Maz'ya. However, the higher-order parabolic ones, with infinitely oscillatory kernels, were not studied in such details. As a basic model, explaining typical difficulties of regularity issues, the $1 \mathrm{D}$ bi-harmonic equation in a domain shrinking to the origin $(0,0)$ is concentrated upon:
\end{abstract}

$$
u_{t}=-u_{x x x x} \quad \text { in } Q_{0}=\{|x|<R(t),-1<t<0\},
$$

where $R(t)>0$ is a smooth function on $[-1,0)$ and $R(t) \rightarrow 0^{+}$as $t \rightarrow 0^{-}$. The zero Dirichlet conditions on the lateral boundary of $Q_{0}$ and bounded initial data are posed:

$$
u=u_{x}=0 \quad \text { at } x= \pm R(t),-1 \leq t<0, \quad \text { and } \quad u(x,-1)=u_{0}(x) .
$$

The boundary point $(0,0)$ is then regular (in Wiener's sense) if $u\left(0,0^{-}\right)=0$ for any data $u_{0}$, and is irregular otherwise. The proposed asymptotic blow-up approach shows that:

(i) for the backward fundamental parabolae with $R(t)=l(-t)^{1 / 4}$, the regularity of its vertex $(0,0)$ depends on the constant $l>0$ : e.g., $l=4$ is regular, while $l=5$ is not;

(ii) for $R(t)=(-t)^{1 / 4} \varphi(-\ln (-t))$ with $\varphi(\tau) \rightarrow+\infty$ as $\tau \rightarrow+\infty$, regularity/irregularity of $(0,0)$ can be expressed in terms of an integral Petrovskii-like (Osgood-Dini) criterion. E.g., after a special "oscillatory cut-off" of the boundary, the function

$$
\tilde{R}(t)=3^{-\frac{3}{4}} 2^{\frac{11}{4}}(-t)^{\frac{1}{4}}[\ln |\ln (-t)|]^{\frac{3}{4}}
$$


belongs to the regular case, while any increase of the constant $3^{-\frac{3}{4}} 2^{\frac{11}{4}}$ therein leads to the irregular one. The results are based on Hermitian spectral theory of the operator $\mathbf{B}^{*}=-D_{y}^{(4)}-\frac{1}{4} y D_{y}$ in $L_{\rho^{*}}^{2}(\mathbb{R})$, where $\rho^{*}(y)=\mathrm{e}^{-a|y|^{4 / 3}}, a=$ constant $\in\left(0,3 \cdot 2^{-\frac{8}{3}}\right)$, together with typical ideas of boundary layers and blow-up matching analysis. Extensions to $2 m$ thorder poly-harmonic equations in $\mathbb{R}^{N}$ and other PDEs are discussed, and a partial survey on regularity/irregularity issues is presented.

Mathematics Subject Classification (2000). 35K55, 35K40.

Keywords. Higher-order parabolic equations, Boundary regularity.

\section{Introduction: Petrovskii's regularity criterion (1934) and extensions}

\subsection{First discussion: regularity as a fundamental issue in potential theory}

Well-posedness of initial-boundary value problems (IBVPs) for linear and nonlinear partial differential equations (PDEs) are key for general PDE theory and applications. The principal question, which was always in the focus of the key research in this area from the nineteenth century to our days, is to determine optimal, and as sharp as possible, conditions on the "shape" of the continuous boundary, under which the solution is continuous at the boundary points. This means that, for a given PDE with sufficiently smooth coefficients, a standard IBVP can be posed with a classical notion of solutions applied.

It is impossible to mention all the cornerstones of such regularity PDE theory achieved in the twentieth century. In the nineteenth century, this study, for the Laplace equation

$$
\Delta u=f \quad \text { in } \Omega \subset \mathbb{R}^{2} \text { or } \mathbb{R}^{3} \quad(0 \in \partial \Omega \text { is the point under scrutiny }),
$$

began by Green (since 1828), Gauss (1940), Lord Kelvin (1847) and Dirichlet himself (in the 1850s), Weierstrass and Neumann (in the 1870s), Hilbert (1899), Schwarz, Poincaré (since 1887); see detailed history of potential theory in Kellogg [39, pp. 277-285]. It is also worth mentioning that classic in potential theory "Lyapunov's surfaces" were introduced about 1898 for general non-convex domains in $\mathbb{R}^{3}$. In the convex case, the problem was already solved in 1870 by Karl Neumann. Lyapunov's study was known to be inspired by Poincaré's earlier papers; a full proof for general Lyapunov surfaces was completed by V.A. Steklov about 1902. As a related issue, as was pointed out by Maz'ya (2008, Private Communication), that Poincaré seems was the first who in the last years of the nineteenth century already knew and used the exterior cone condition for the regularity of boundary points. As is well known from his private letters, discussions with several Russian mathematicians, including e.g., his supervisor 
P.L. Chebyshov ${ }^{1}$ and Steklov, Lyapunov was constantly rather anxious studying new and amazing but not fully rigorously justified ideas and methods by Poincaré. Possibly, this led him to develop the concept of "Lyapunov's surfaces" in potential theory about that time. Indeed, this is related to some of other fundamental concepts in his stability theory, where discussions with Poincaré were also known to take place.

For the second-order Laplace equation (1.1) and the heat equation

$u_{t}=\Delta u \quad$ in $Q, \quad(0,0) \in \partial Q$ is characteristic, where $\partial Q$ is tangent to $\{t=0\}$,

regularity theory was almost fully completed in the 1920-1930s. These various ideas and key results on optimal regularity criteria created the amazing history, which is explained in a number of classic monographs. For convenience, we will begin our discussion with some historical aspects in the next Sect. 3, where we hope to present some new and not that well-known regularity features and peculiarities for the sake of the attentive Reader.

The present paper uses the asymptotic blow-up approach from reactiondiffusion theory to such optimal boundary regularity questions. It is shown that, for a number of higher-order PDEs with essentially oscillatory kernels, these can be treated by blow-up evolution via approaching a "singular" boundary point. Surely, this is not a principal novelty not only for the heat equation (1.2) [73], but also in elliptic regularity theory, where, together with other techniques, rescaled linear operator pencils were shown to be key; we refer to Kozlov-Maz'yaRossmann's monographs [47,48] and surveys in [24,45,63,87] for details and full history. In this connection, Kondrat'ev's seminal paper [44] for higher-order linear elliptic equations ${ }^{2}$ (see Kondrat'ev-Oleinik's very detailed survey of 1983 [45] for up to the 1980s and the most recent monograph [11] for a huge number of more modern extensions), thought not devoted to the regularity issues, represented a novel and involved use of spectral properties of operator pencils for describing the asymptotics of solutions near corner singularities. This analysis assumes a kind of an "elliptic evolution" approach for elliptic problems, which then is not well-posed in Hadamard's sense but can trace out the behaviour of necessary global orbits that approach the singularity point. For nonlinear elliptic equations, these ideas got various developments and diversions since the 1970s; see Véron's monographs $[84,85]$ and [9] for further history and key references.

\footnotetext{
${ }^{1}$ A.M. Lyapunov Master's Thesis "On Stability of Spheroidal Equilibrium Forms of a Rotating Fluid", written under the supervision of Chebyshov, was defended in the S.-Petersburg University, 27th January, 1885.

${ }^{2}$ As explained in [44], previous results on normal solvability of elliptic boundary value problems in domains with angular (in $\mathbb{R}^{2}$ ) and conical $\left(\mathbb{R}^{N}, N \geq 3\right.$ ) points were obtained by Fufaev ( $m=1, N=2,1960)$, Volkov (1962), Birman and Skvortsov (1962), Lopatinskii (1963). Earlier Nikol'skii's results [72] in the 1956-1958 on boundary differential properties of functions in the Nikol'skii spaces $H_{p}^{r}$ defined on regions in $\mathbb{R}^{2}$ with angular points and hence criteria of such a regularity for the Laplace equation should be mentioned.
} 
Classic Wiener's and operator pencil ideas were successfully developed for many higher-order elliptic equations with a typical representative:

$$
(-\Delta)^{m} u=f \quad \text { in } \Omega \subset \mathbb{R}^{N} \quad(m \geq 2),
$$

in the papers by Maz'ya with collaborators since the 1970s; see e.g., [65] and $[47,48,62,63]$ as a guide. Though regularity point analysis for (1.3) revealed a lot of new phenomena and mathematical difficulties relative the classic ones for $m=1$ (see comments later on), there are many papers on this subject, where essential progress was achieved, especially in the recent ten years.

\subsection{Higher-order parabolic PDEs: on some known regularity results}

For 2 mth-order parabolic equations, where the basic model is the canonical polyharmonic equation

$$
u_{t}=-(-\Delta)^{m} u, \quad \text { with } m \geq 2,
$$

regularity questions have been also addressed in the literature, however essentially less actively than for elliptic PDEs referred to above. Among results of classic solvability theory, which can be found in a number of monographs on linear and nonlinear equations, systematic approaches to the study of non-cylindrical domains with a characteristic vertex $(0,0)$, where ${ }^{3}$ the tangent plane $\{t=0\}$ is horizontal in the $\{x, t\}$-space appeared already in the 1960s.

Mihailov papers in 1961 and 1963 [66-68] treated the case, where the parabolic boundary of the domain $Q_{0}$ lies below the characteristic plane $t=0$ and has a typical form of the corresponding "backward" paraboloid of the fundamental solution $^{4}$. In $[67,68]$, this shape is perturbed by factor that makes the paraboloid "sharper", i.e., becomes "more regular"; see further comments on that.

It is worth mentioning that, almost simultaneously with the fundamental "elliptic" paper [44], Kondrat'ev in 1966 published another key "parabolic" paper [43], which is less known. ${ }^{5}$ Here, Kondrat'ev dealt with the singularity issues

\footnotetext{
${ }^{3}$ Here and later on, we indicate only the results that are related to our blow-up setting to be explained shortly; these deep papers contain other involved conclusions.

${ }^{4}$ Q.v. more recent Mihailov's research on existence of boundary values of poly-harmonic functions for domains with smooth boundary; see references in [69].

${ }^{5}$ It is useful to compare the total number of citations in the MathSciNet (February 2009): the elliptic paper [44] has the record 240 citations, while the parabolic one [43] has eight citations, i.e., 30 times less! However, the proposed scaling techniques $[43,44]$ leading to operator pencils are almost identical in both parabolic and elliptic papers. Moreover, the parabolic pencil analysis [43] directly applies to some ultraelliptic PDEs. The asymptotic expansions derived by spectral theory near the vertex 0 ,

$$
u(x, t) \sim \sum_{(j, k)} \tau^{-\mathrm{i} \lambda_{j} / 2 m}(\ln \tau)^{k} \psi_{k j} \quad\left(\lambda_{j} \text { are eigenvalues of pencils }\right)
$$

are similar for the elliptic $(\tau=|x|)$ and parabolic $(\tau=-t)$ cases. Here, the $\ln ^{k} \tau, k=0,1, \ldots, k_{j}$ multipliers reflect finite algebraic multiplicities $k_{j}$ of $\lambda_{j}$ with associated generalized $C^{\infty}$ eigenvectors $\left\{\psi_{k j}\right\}$. Note that, even for the heat equation, the required pencil spectral properties are not that easy to get [4].
} 
for more general than (1.4) parabolic equations and various boundary conditions on a fixed fundamental paraboloid. Complicated asymptotics of solutions near such singular points were obtained, and, as a result, a solvability criterion was proved for existence of weak solutions in special weighted Sobolev-Slobodetskiitype spaces. Concerning other related earlier papers on parabolic PDEs in noncylindrical domains, Solonnikov [80] and Ivasishen [35] should be mentioned.

In 1971, Feigin [22] extended the results to the case when, in any neighbourhood of the vertex $(0,0)$, the boundary lies on both sides of the characteristic plane $\{t=0\}$ (see [78] for Feigin's contribution to elliptic regularity theory in the 1970s). These deep results initiated further subsequent study, which nevertheless was not that exhaustive and sharp as in elliptic theory. We will try to explain why (the answer seems easy: this lifts the regularity problem to another level of difficulty). The current standing of this regularity parabolic theory can be traced out further by the MathSciNet, but the author reports that getting a clear convincing view is not easy.

\subsection{Main goal: deriving sharp asymptotics of solutions near vertex depending on boundary geometry}

As a consequence of the above discussion involving a number of strong previous results, optimal conditions of regularity/irregularity of the vertex $(0,0)$ for the poly-harmonic flow (1.4) obviously demand the following:

Goal I: sharp asymptotic expansion of solutions near $(0,0)$ are necessary for rather arbitrary backward characteristic (non-fundamental) paraboloids to get regularity of the vertex via the geometry of the boundary nearby.

In other words, we check whether it is possible to characterize a sharp asymptotic behaviour of classic (strong) solutions near the characteristic vertex, when the paraboloid is not given by the backward variable of the fundamental solution, i.e., can take various forms. Loosely speaking, we are going to mimic a Petrovskiitype criterion for higher-order parabolic equations such as (1.4). We must admit that, literally, this goal is not and cannot be achieved literally, but a partial first step is indeed doable. Once such an asymptotic has been obtained, one can reevaluate the solution near the vertex in any necessary weighted metric, eventually to check whether solution exists (is continuous at the vertex) in weaker sense.

As usual, we find useful, in order to characterize our blow-up approach, to consider first the simplest 1D bi-harmonic equation

$$
u_{t}=-u_{x x x x} \quad(m=2),
$$

which even in the one $x$-dimension provides us with a number of not that mathematically pleasant surprises. It seems that the main difference (and the origin of extra difficulties) between the higher-order elliptic case (1.3) and the parabolic one (1.7) is that the later has always oscillatory kernel of changing sign infinitely many times, while for (1.3), the kernel is less oscillatory and even remains positive 
in some dimensions even for $m \geq 2$. As is well known since Maz'ya-Nazarov's results for $m=2, N \geq 8$ in 1986 [64], the changing sign kernels of elliptic operators produce new phenomena, where classic regularity techniques may fail and even standard conical points can be irregular and even singular, i.e., solutions unbounded at the vertex. Note that Kondrat'ev's sharp estimates such as (1.5) [44] can also indicate such a possibility, if sharp bounds on first pencil's eigenvalues are known. Therefore, a simple 1D model (1.7) becomes a key parabolic PDE with such an infinitely oscillatory kernel, so firstly we concentrate on this equation in what follows.

As a unified issue of the present analysis of higher-order parabolic and other PDEs (i.e., without any order-preserving or positive kernel features), we aim a sharp treatment of boundary regularity conditions by using spectral theory of related rescaled non self-adjoint operators or pencils of such operators. A full justification of some "approximate" regularity conditions are very difficult and lead to open problems. As an independent feature of the regularity questions, which underlines their difficult nature, as far as we know, even for the simplest higherorder elliptic or parabolic operators with real constant coefficients, there is a clear and natural difficulty for determining sufficiently sharp regularity conditions for linear operators with kernels of essentially changing sign. For such operators, Wiener's and other related capacity-like techniques, which assume kernel positivity, are often and naturally expected to fail, so new techniques are necessary.

Of course, we exclude those operators for which the regularity is guaranteed by standard embedding results, or when the irregularity is induced by some symplectic-Hamiltonian properties, which do not allow any shrinking of the domain under consideration. E.g., the latter is true for Schrödinger operators ${ }^{6}$ i $D_{t}+(-\Delta)^{m}$, where $L_{x}^{2}$-norm is typically preserved.

Thus, our goal is to develop an asymptotic method of blow-up regularity analysis, firstly, for the simplest 1D bi-harmonic equation, and next extend to poly-harmonic ones (1.4). In general, together with other examples of PDEs, we follow the principle (as we have seen, such kernels have been studied much less in the mathematical literature)

Goal II: to deal with kernels having infinitely many sign changes,

that can occur not necessarily for parabolic PDEs only. We will show that a kind of Petrovskii-like integral "criterion" for regularity/irregularity of boundary points $[74,75](1934-1935)$ can be visualized. However, for operators with oscillatory kernels of changing sign, a standard deterministic analysis of such integral Osgood-Dini-type conditions via divergence/convergence (as for the second-order or other operators with more positively dominant kernels) is not available. Secondly, we discuss possible extensions of the method to other types of linear and nonlinear PDEs with the kernels like (1.8). Meantime, we continue our survey of the history of crucial for us regularity issues and integral criteria.

${ }^{6}$ The author thanks I.V. Kamotski for a discussion that clarified this property. 


\section{Elliptic equations as the origin of regularity theory: Zaremba [88], Lebesgue [53], and Wiener [86]}

\subsection{Laplace equation: early days of existence and nonexistence theory}

The first criterion (a necessary and sufficient condition) of regularity of a boundary point 0 was the Wiener famous one for the Dirichlet problem for the Laplace equation

$\Delta u=f$ in a bounded domain $\Omega \subset \mathbb{R}^{2}, 0 \in \partial \Omega \quad(u=0$ on $\partial \Omega$, for definiteness $)$, derived in 1924 [86]. The case of arbitrary $N \geq 2$ was also embraced. It is formulated in terms of a diverging series of capacities (measuring the thickness of the complement of $\Omega$ near 0 ) of a discrete family of domains shrinking to the given point 0 on the boundary; see extra details and further discussions in CourantHilbert [13, p. 306] and in Kellogg [39, p. 330].

Concerning nonexistence for the Dirichlet problem (see [47,48,61] for details), the first rigorous nonexistence example belonged to Stanislav Zaremba in 1911 [88, p. 310]. He dealt with a plane domain whose boundary has isolated points, not connected; e.g., the surface comprised a sphere and its centre; see comments in $[39$, p. 285]. The key non-solvability example was constructed by Lebesgue in $1913[53,54]^{7}$ who discovered striking examples of irregularity. E.g., he showed that, for a domain in $\mathbb{R}^{3}$ with the inner cone obtained by rotating the function $x_{2}=\mathrm{e}^{-1 / x_{1}}$ for $x_{1}>0$ small, about the $x_{1}$-axis (then the points with $x_{2}>\mathrm{e}^{-1 / x_{1}}$ do not belong to the domain, i.e., the domain has a sharp cusp turned inwards, a Lebesgue spine), the origin 0 is irregular. Figuratively speaking, despite such a thin spine connection of the 0 with the boundary, "effectively" the centre remains "separated" reminding earlier Zaremba's nonexistence construction. The same nonexistence occurs for the shape $x_{2}=x_{1}^{\left|\ln x_{1}\right|^{\varepsilon}}$, with any $\varepsilon>0$. However, 0 is regular if it can be touched from outside by any cone given by rotation of $x_{2}=x_{1}^{k}$ for any $k>0$ fixed; see comments in Petrovskii's PDE text book [76, p. 325]. Carleman's PhD Thesis [12] of 1916 was also one of the first study of singularities of solutions of elliptic PDEs at non-regular boundary points; see also [45, Introd.].

Forty years later, new examples of nonexistence, following ZarembaLebesgue-Uryson's ideas (see below about Uryson's contribution to nonexistence), were developed by Kondrat'ev [42, p. 129]. For the Dirichlet problem $\Delta u+u=0$ in $\Omega$, he produced further examples of non-solvability for not simply connected domains for $N=2$ (sufficient number of disjoint small discs are removed), and domains in $\mathbb{R}^{3}$ such as a ball with line segments or narrow cylinders removed to get sufficiently small interior diameter (one end stays on the surface for not violating connectedness). These constructions extend to arbitrary $N$ and $2 m$, including

\footnotetext{
${ }^{7} \mathrm{~A}$ full Lebesgue account on the Dirichlet problem includes his important earlier paper [51], where the variational method was employed in 2D to solving under very general conditions, and another key one [52], where "barrier" (in Poincaré's terminology) techniques were employed; $[54]$ is his final summarizing all key ideas and results.
} 
the most delicate range $N>2 m$. By an $m$-capacity technique, [42] also studied the question of $k$-regularity $(k \leq m)$ at a boundary point $A$ meaning that any $u \in W_{m, 0}^{2}$ has $k-1$ derivatives vanishing there asymptotically, i.e., up to a set of the measure $o\left(a^{N}\right)$ in $|x-A|<a$ as $a \rightarrow 0$.

It is also worth mentioning further and later results on the Dirichlet problem obtained by Uryson in the beginning of 1920s [5,82] (nonexistence of Lebesgue's type) and Keldysh in 1941 [37,38] (stability relative domain perturbations, where the notion of "Keldysh balls" was invented). ${ }^{8}$ We refer to a more detailed survey in the earlier preprint [27, Sect. 2.2].

\subsection{On some recent achievements}

We are not going to deal with elliptic equations in what follows. However, for the sake of a further comparison with "parabolic" results, it is worth mentioning that a full extension of Wiener's regularity test (criterion) via the concepts of potentialtheoretic Bessel (Riesz) capacities to $2 m$ th-order strongly elliptic equations with real constant coefficients,

$$
\left\{\begin{array}{l}
L(\partial) u=f, \quad f \in C_{0}^{\infty}(\Omega), \quad u \in H_{0}^{m}, \\
L(\partial)=\sum_{|\alpha|=|\beta|=m} a_{\alpha \beta} \partial^{\alpha+\beta}, a_{\alpha \beta} \equiv a_{\beta \alpha}, \quad(-1)^{m} L(\xi)>0 \text { in } \mathbb{R}^{N} \backslash\{0\},
\end{array}\right.
$$

was completed in the case $N=2 m$ in 2002 by Maz'ya. ${ }^{9}$ The case $N>2 m$ also admits a treatment, though applied for a subclass of the so-called strongly elliptic equations. For $N \leq 2 m-1$, the point is always regular by the classic Sobolev embeddings; see $[62,63]$ and references therein to earlier results and extensions.

Concerning the related questions of sharp asymptotic behaviour near Lipschitz boundary at 0 for problems (2.1), in [46], a rather general case of the boundary given by

$$
\Omega=\left\{x=\left(x^{\prime}, x_{n}\right) \in \mathbb{R}^{N}: \quad x_{n}>\varphi\left(x^{\prime}\right)\right\}, \quad \varphi(0)=0,
$$

is studied. The asymptotics as $x \rightarrow 0$ of a class of solutions are shown to depend on the Lipschitz constant of $\varphi\left(y^{\prime}\right)$ in a neighbourhood of 0 :

$$
\kappa(\rho)=\sup _{\left|y^{\prime}\right|<\rho}\left|\nabla \varphi\left(y^{\prime}\right)\right| \quad\left(\text { estimates are simplified if } \int_{0} \frac{\kappa^{2}(\rho)}{\rho} \mathrm{d} \rho<\infty\right) .
$$

Among others, let us also note an unusual Wiener's-type regularity and existence result for blow-up on the boundary solutions of the semilinear elliptic equation

$$
\Delta u-|u|^{q-1} u=0 \quad \text { in } \Omega, \quad u(x) \rightarrow+\infty \quad \text { as } x \rightarrow \partial \Omega \quad(q>1) ;
$$

\footnotetext{
${ }^{8}$ Note a curious aspect: geographically, both these contributions to potential theory were related to the same place: the Miusskaya Square in Moscow, Russia, which somehow remained to be a PDE centre for a half of century to come.

${ }^{9}$ The principal extension of Wiener's-like capacity regularity test to the nonlinear degenerate $p$-Laplacian operator $-\Delta_{p} u \equiv-\nabla \cdot\left(|\nabla u|^{p-2} \nabla u\right), p \in(1, N]$, was also due to Maz'ya in 1970 [59]; later on, his sufficient capacity regularity condition turned out to be optimal for any $p>1$.
} 
see $[49,57]$, where a long list of other related references is presented (Eq. (2.2) will be cited again).

\section{Parabolic PDEs: Petrovskii's $\sqrt{\log \log }$ criterion (1934) and around}

\subsection{Parabolic regularity theory began with the heat equation: first results and definitions}

From the beginning, for the second-order parabolic equations, the boundary point regularity analysis took a different direction (i.e., not of Wiener's type). This was Petrowsky $[74,75]$, who in $1934^{10}$ completed the study of the regularity question for the $1 \mathrm{D}$ and 2D heat equation in a non-cylindrical domain. For further use, we formulate his result in a blow-up manner, which in fact was already used by Petrovskii in 1934 [74]. This is about the question on a irregular or regular point $(x, t)=(0,0)$ for nonnegative solutions of the IBVP

$$
\left\{\begin{array}{l}
u_{t}=u_{x x} \quad \text { in } Q_{0}=\{|x|<R(t),-1<t<0\}, \quad R(t) \rightarrow 0^{+} \text {as } t \rightarrow 0^{-}, \\
\text {with bounded smooth data } u(x, 0)=u_{0}(x) \geq 0 \quad \text { on }[-R(-1), R(-1)] .
\end{array}\right.
$$

Here the lateral boundary $\{x= \pm R(t), t \in[-1,0)\}$ is given by a function $R(t)$ that is assumed to be positive and $C^{1}$-smooth for all $-1 \leq t<0$ and is allowed to have a singularity of $R^{\prime}(t)$ at $t=0^{-}$only. We then study the value of $u(x, t)$ at the end "blow-up" characteristic point $\left(0,0^{-}\right)$, to which the domain $Q_{0}$ is said to "shrink" as $t \rightarrow 0^{-}$.

For the heat equation, the first existence of a classical solution result was obtained by Gevrey in 1913-1914 [32] (see Petrovskii's references in [74, p. 55] and [75, p. 425]), which assumed that the Hölder exponent of $R(t)$ is larger than $\frac{1}{2}$. In our setting, at $t=0^{-}$, this comprises all types of boundaries given by the functions:

$$
R(t)=(-t)^{\nu} \quad \text { with any } \nu>\frac{1}{2} \text { are regular (Gevrey [32]). }
$$

Note that, for $2 m$ th-order parabolic equations, a similar result saying that, for

$$
R(t)=(-t)^{\frac{1}{2 m}}, \quad \text { the problem is uniquely solvable }
$$

(in Slobodetskii-Sobolev classes, i.e., not in the classic sense) was proved by Mihăllov [66] almost 60 years later.

\footnotetext{
${ }^{10}$ Actually, this research was performed earlier: his most famous 1935 paper in Compositio Mathematica [75] was submitted for publication "(Eingegangen den 27. November 1933.)" [75, p. 419]. Before, the results were discussed in: "Diskussion im wahrscheinlichkeits-theoretischen Seminar der Universität Moskau", which led to Kolmogorov's problem solved by Petrovskii in [75, p. 414]; see Abdulla [2,3] for further details concerning this fundamental problem called nowadays Kolmogorov-Petrovskii's one.
} 
Regular point: As usual in potential theory, the point $(x, t)=(0,0)$ is called regular (in Wiener's sense, see [62]; sometimes is also called boundary) if any value of the solution $u(x, t)$ can be prescribed there by continuity as a standard boundary value on $\partial Q_{0}$. In particular, as a convenient and key for us evolution illustration, $(0,0)$ is regular if the continuity holds for any initial data $u_{0}(x)$ in the following sense:

$u=0$ at the lateral boundary $\{|x|=R(t),-1 \leq t<0\} \Longrightarrow u\left(0,0^{-}\right)=0$.

Irregular point: Otherwise, the point is irregular (or inner) if the value $u\left(0,0^{-}\right)$is not arbitrary and is given by the parabolic evolution as $t \rightarrow 0^{-}$. Hence, formally, $(0,0)$ does not belong to the parabolic boundary of $Q_{0}$, i.e., is inner in a natural sense.

\subsection{On some details of Petrovskii's analysis in 1934-1935 and extensions}

Reading last twenty five or so years various papers related to regularity issues for parabolic PDEs, the author found several and sometimes rather distinctive treatments of Petrovskii's results. For instance, his 1934 paper [74] was not cited practically in no such papers, but the one [4]. There were also discrepancies in treating whether or not Petrovskii derived the integral Osgood-Dini-like conditions (surely, he did already in 1934). Therefore, it seems reasonable to mention here some of these aspects of Petrovskii's original derivation of his celebrated criterion.

Thus, according to (3.1), in both the cases of regularity/irregularity, there occur interesting asymptotic problems of the behaviour of $u(x, t)$ in $Q_{0}$ as $t \rightarrow 0^{-}$, which are of singular (finite-time blow-up) type. These problems were solved by Petrovskii $[74,75]$ in $1934-1935$, who derived a regularity criterion ${ }^{11}$ for (3.1) by constructing rather tricky sub- and super-solutions of the heat equation. These are "subparabolish" and "superparabolish" ones in the original Petrovskii terminology in German, [75, p. 386].

For instance, to prove irregularity for the characteristic curve at $\left(0,0^{-}\right)$

$$
x^{2}=4(1+\varepsilon)|t| \ln |\ln | t||, \quad \varepsilon>0,
$$

he introduced the following explicit sub-solution "Barriere der Irregularität" [74, p. 58], [75, p. 394] (see also comments in [70]):

$$
v(x, t)=\frac{-1}{|\ln | t||^{1+\varepsilon_{1}}} e^{\frac{-x^{2}}{4 t} k}+\frac{1}{\ln |\ln | t||} \quad\left(v_{t}<v_{x x}\right), \quad \text { where } \frac{1+\varepsilon_{1}}{k}<1+\varepsilon,
$$

and $\varepsilon_{1}<1, k<1$ are some positive constants. Vice versa, to prove regularity for

$$
x^{2}=4|t| \ln |\ln | t||, \quad(\text { i.e., } \varepsilon=0),
$$

\footnotetext{
${ }^{11}$ Cf. quoting these necessary and sufficient conditions in Kondrat'ev [43, p. 450].
} 
the following explicit super-solution "Barriere der Regularität" [74, p. 58] was used:

$$
v(x, t)=\frac{-1}{\left.2|\ln | t\right|^{1 \frac{1}{6}}} e^{\frac{-x^{2}}{4 t}}+\frac{1}{\sqrt[6]{|\ln | t||}} \quad\left(v_{t}>v_{x x}\right) .
$$

In [75], the standard nowadays notation $\underline{u}(x, t)$ and $\bar{u}(x, t)$ for sub- and supersolutions were used. For more general boundaries given by

$$
x^{2}<4 t \ln \rho(t)
$$

Petrovskii [75, p. 393] introduced the related function $\varphi(t)$ determined from

$$
\int_{t_{0}}^{t} \frac{\rho(\eta) d \eta}{\eta}=6 \log \varphi(t)
$$

which was used in constructing suitable super- and sub-solutions. Here, (3.8) is the origin of Petrovskii's criterion (3.11) given below.

In his another paper [73] in 1934, Petrovskii also applied his novel method of sub- and super-solutions. In other words, this has been done before the classic Nagumo, or Müller-Nagumo-Westphal, Lemmas from the 1940-50s. Note that, for the Dirichlet problem, barrier-type ideas in the méthode de balayage, or sweeping out, were used by Poincaré as early as in 1887 [77], with further development by Lebesgue in 1912 [52] and by Perron in 1923 for $\Delta u=0$ [73, p. 432]. Now, Petrovskii used these barrier approaches in the study of randomness that had a great influence on random processes theory, which he connected with linear elliptic equations. This role was first explained in Khinchin's monographs in 1933 and 1936 [40]; see below. Note also that, in [73], Petrovskii dealt with the Dirichlet problems ("Dirichletschen Problems") for elliptic equations such as [73, p. 428]

$$
M_{x x} \frac{\partial^{2} u}{\partial x^{2}}+2 M_{x y} \frac{\partial^{2} u}{\partial x \partial y}+M_{y y} \frac{\partial^{2} u}{\partial y^{2}}+2 M_{x} \frac{\partial u}{\partial x}+2 M_{y} \frac{\partial u}{\partial y}=0,
$$

where " $M_{x x} M_{y y} \geq M_{x y}^{2}$ ", and, surely, Petrovskii's typical double $\ln \ln$ appeared as an its solution " $u=\ln \ln \frac{1}{\sqrt{x^{2}+y^{2}}}$ " on pp. 437-438. In three years time, sub/supersolution techniques were applied in the seminal KPP-paper, 1937 [41].

Petrovskii's 1934's paper [74] is fully devoted to the optimal regularity and existence-uniqueness analysis for the heat equation, where he already introduced his $2 \sqrt{\ln l n}$-criterion including the converging/diverging integral as in (3.11) for irregularity/regularity. For instance, he showed that the "5-log curve" and so on $[74$, p. 57] (cf. [75, p. 404])

$x^{2}=4|t|\left[\ln |\ln t|\left|+1 \frac{1}{2} \ln \ln \right| \ln |t||+\ln \ln | \ln |t||(1+\varepsilon) \ln \ln \ln \ln | \ln |t| \mid\right] \quad$ u. s. w.

is regular for $\varepsilon \leq 0$ and is irregular for any $\varepsilon>0$. In addition, for the $2 \mathrm{D}$ heat equation

$$
u_{t}=u_{x x}+u_{y y}
$$


it is mentioned $[74$, p. 59] that the regularity/irregularity issues occur about the surface

$$
x^{2}+y^{2}=-4(1+\varepsilon) t \ln |\ln | t|| .
$$

In particular, then [75, p. 397] the super-solution took the same form (3.5), where $x^{2}$ is replaced by $x^{2}+y^{2}$. In the later paper [75] in 1935 , extensions to the heat equation in $\mathbb{R}^{k} \times \mathbb{R}_{+}$were mentioned.

Hence, we attribute Petrovskii's criterion to 1934 [74], and not to 1935, [75], as used had been done in most of other "parabolic" regularity papers, we have referred to here.

Thus, using such novel barriers, Petrovskii [74,75] established the following:

(i) $R(t)=2 \sqrt{-t} \sqrt{\ln |\ln (-t)|} \Longrightarrow(0,0)$ is regular, and

(ii) $R(t)=2(1+\varepsilon) \sqrt{-t} \sqrt{\ln |\ln (-t)|}, \varepsilon>0 \Longrightarrow(0,0)$ is irregular.

More precisely, he showed that, for the curve expressed in terms of a positive function $\rho(h) \rightarrow 0^{+}$as $h \rightarrow 0^{+}\left(\rho(h) \sim \frac{1}{|\ln h|}\right.$ is about right) as follows:

$$
R(t)=2 \sqrt{-t} \sqrt{-\ln \rho(-t)},
$$

the sharp regularity criterion holds:

$$
\int_{0} \frac{\rho(h) \sqrt{|\ln \rho(h)|}}{h} \mathrm{~d} h<(=)+\infty \Longrightarrow\left(0,0^{-}\right) \text {is irregular (regular). }
$$

It is worth underlying again that both converging (irregularity) and diverging (regularity) integrals in (3.11) as Dini-Osgood-type regularity criteria already appeared in the first Petrovskii paper [74, p. 56] of 1934. It should be mentioned that, since Petrovskii used proposed him approach of constructing super- and sub-solutions, solving such partial differential inequalities led (as usual) unavoidably to a technical assumptions. For instance, for the super-solution (regularity of $(0,0))$, this is $[75$, p. 392$]$

$$
\text { 2. } \quad t \log \rho(t) \rightarrow_{t \rightarrow 0} 0 ;
$$

and, for the sub-solution (irregularity of $(0,0))$ [75, p. 397],

$$
\begin{array}{ll}
\text { 3. } & t \log \rho(t) \rightarrow 0 \quad \text { für } t \rightarrow 0 ; \quad \text { and } \\
\text { 5. } & \text { es ist } \quad \frac{\rho^{\prime}(t)}{\rho(t)} \leq \frac{1}{8|t|} .
\end{array}
$$

These purely technical assumptions can be got rid of [1]; our blow-up spectralboundary layer approach is also assumptionless and rigorous for the heat equation, see Sect. 7.7.

The results of $[74,75]$ were key important for probability theory, where this result is expressed as follows: if $x_{t}$ is a 1D Wiener process and $\Phi(t)>0$ is monotone such that

$$
\int^{\infty} \frac{\Phi(t)}{t} \mathrm{e}^{-\Phi^{2}(t) / 2} \mathrm{~d} t=\infty
$$


then, with unit probability, $\left|x_{t}\right| \leq \Phi(t)$ for all $t \gg 1$. On the contrary (this is about the criterion), if the integral converges, then with unit probability $\exists\left\{t_{k}\right\} \rightarrow \infty$ such that $\left|x_{t_{k}}\right|>\Phi\left(t_{k}\right)$; an optimal extension was done in [40]. Eventually, these Petrovskii's results led to the so-called the Law of Iterated Logarithms (LIL) [14, p. 392], which was discovered by Khinchin and Kolmogorov. Earlier, in 1924 Khinchin proved that, for a sequence $\left\{X_{i}\right\}_{i \geq 1}$ of independent random variables with values \pm 1 with the probability $\frac{1}{2}$, there holds:

$$
S_{n} \equiv X_{1}+X_{2}+\cdots+X_{n} \leq \sqrt{(2+\varepsilon) n \ln \ln n} \text { for all } n \gg 1(\forall \varepsilon>0)
$$

with the probability one. This sharp estimate improved earlier Hausdorff's inequality (1913) $S_{n}=o\left(n^{\frac{1}{2}+\varepsilon}\right)$, Hardy-Littlewood's one (1914) $S_{n} \leq C \sqrt{n \ln n}$, and Steinhaus' inequality (1922) $\limsup S_{n} / \sqrt{2 n \ln n} \leq 1$. As a final step, in 1929, Kolmogorov proved the LIL for any bounded independent random variables not assuming that the summands were identically distributed; see more details in [14, Chap. 7] and [33]. Clearly, (3.9) has probabilistic roots in (3.13).

A Wiener-type criterion, already established by Khinchin [40] (an earlier result was in 1933) in a probability representation, for the boundary regularity for problems such as (3.1) and in $\mathbb{R}^{N}$, was derived by Landis in 1969 [50]. This was done in terms of converging/diverging series of potentials of shrinking sets involved. See also [20] for a criterion via thermal capacity also obtained along the lines of derivation of Wiener's one for Laplace's equation. These results were not stated in the blow-up evolution and more "practical" Petrovskii's style (3.11), though Wiener's-type capacity criteria serve for more general types of boundaries than according to Petrovskii's approach.

Petrovskii's integral criterion of the Dini-Osgood type given in (3.11) is true in the $N$-dimensional radial case with (see [1]-[3] for the recent updating)

$$
\sqrt{|\ln p(h)|} \quad \text { replaced by }|\ln p(h)|^{\frac{N}{2}} \text {. }
$$

Several boundary regularity/irregularity results are now known for a number of quasilinear parabolic equations including degenerate porous medium operators. However, some difficult questions remain open even for the second-order parabolic equations with order-preserving semigroups. We refer to $[1-3,34,56,70]$ as a guide to a full history and the extensions of these important results.

As far as we know, (3.9) is the first clear appearance of the "magic" $\sqrt{\log \log }$ in PDE theory, currently associated with the "blow-up behaviour" of the domain $Q_{0}$ and corresponding solutions. Concerning other classes of nonlinear PDEs generating blow-up $\sqrt{\log \log }$ in other settings, see references in [28].

Finally, as an introduction to difficult features of higher-order parabolic regularity to be studied, it is worth clearly stating that

for solutions of changing sign of the HE, Petrovskii's criterion (3.11) fails. 
Obviously, positive subsolutions to prove irregularity are not applicable, while the regularity can be still proved by comparison. In fact, a general criterion for arbitrary bounded changing sign data $u_{0}(x)$ in (3.1) cannot be derived in principle. The origin of this is the same for the HE (3.1), the bi-harmonic one (1.7), and others; see our analysis below.

\subsection{Example: a refined asymptotics for the porous medium equation}

In connection with the blow-up log-log, it is worth mentioning the asymptotic result (seems, unique of this type) for the Dirichlet problem as in (3.1) [34, Sect. 2], which is now formulated for the radial porous medium equation in the pressure form:

$$
\begin{gathered}
v_{t}=\Delta v^{m}, \quad m>1, \quad \text { where the pressure } u=v^{m-1} \text { solves: } \\
u_{t}=u \Delta u+\gamma|\nabla u|^{2} \quad \text { in } Q_{0}, \quad \text { with } R(t)=\sqrt{-t}, \quad \gamma=\frac{1}{m-1}>0 .
\end{gathered}
$$

Then the $\log -\log$ occurs in the asymptotic behaviour of nonnegative solutions as $t \rightarrow 0^{-}$:

$$
u(x, t) \sim \frac{1}{4 \ln |\ln (-t)|} \rightarrow 0 \quad \text { uniformly on subsets } \quad\left|\frac{|x|}{\sqrt{-t}}-1\right| \gg \frac{1}{\ln |\ln (-t)|}
$$

The computations leading to (3.16) by asymptotic-matching ideas (some will be involved in our further study) are not easy, and a complete proof was not supplied in [34]. Regardless the comment at the end of page 4 in [34], it seems that a full and entirely rigorous justification of this kind of matching with "floating" matching point can be extremely difficult and even seems illusive. In fact, this emphasizes a general complexity of such type of results even for second-order parabolic PDEs with the Maximum Principle.

Remark: on boundary criterion for (3.15). Obviously, the delicate asymptotics (3.16) allows one to detect the actual "criterion" of the boundary regularity for the Eq. (3.15) (strangely, this was not addressed in [34]). We use the scaling invariance of (3.15):

$$
u=A \hat{u}, \quad x=\sqrt{A} \hat{x}, \quad \text { where } A>0 \text { is an arbitrary constant. }
$$

According to natural concepts of approximate similarity solutions (see e.g., [79, Chap. 6]), we next assume that

$$
A=A(t) \text { is not a constant, but a slow decaying function (relative to } \sqrt{-t} \text { ). }
$$

Then the equation for $\hat{u}(\hat{x}, t)$ will include an extra very small asymptotic perturbation, which will not affect the asymptotics. Finally, according to (3.16) we set

$$
A(t)=\frac{1}{4 \ln |\ln (-t)|}, \quad \text { so that } \hat{u} \sim 1
$$


on the corresponding compact subsets as $t \rightarrow 0^{-}$. Thus, $u(0,0) \neq 0$ for the function

$$
\hat{R}(t) \sim 2 \sqrt{-t} \sqrt{\ln |\ln (-t)|}
$$

and, in this irregularity condition, Petrovskii's magic $\sqrt{\ln \ln }$ again occurs. The constant " 2 " here seems not relevant as in (3.9), since (3.15) is nonlinear and admits also the symmetry $u \mapsto A u, \quad t \mapsto \frac{t}{A} \quad$ for any $A>0$, so that the domain behaviour with $\hat{R}(t) \sim \frac{2}{\sqrt{A}} \sqrt{-t} \sqrt{\ln |\ln (-t)|}$ is irregular for $(0,0)$.

Thus, since the $1930 \mathrm{~s}$, Petrovskii's regularity $\sqrt{\log \log }$-factor entered parabolic theory and generated new types of asymptotic blow-up problems, which have been solved for a wide class of parabolic equations with variable coefficients as well as for some quasilinear ones. Nevertheless, such asymptotic problems are very delicate and some of them of Petrovskii's type remain open even in the second-order case.

For higher-order poly-harmonic operators, the situation becomes much more difficult, since the Maximum and Comparison Principles and order-preserving properties fail, so classic barrier techniques associated with sub-, super-, and other solutions are not applicable in principle. This precisely reflects the area of the present research: no Maximum Principle and any barrier tools, so that the boundary regularity problem in Petrovskii's setting falls into the scope of a blow-up asymptotic behaviour study.

\section{Towards Petrovskii's criterion for the bi-harmonic flow: asymptotic blow-up setting, method, and layout of the paper}

\subsection{The basic Dirichlet problem under consideration}

As a basic model, bearing in mind a "minimal" extension of the heat equation (3.1) by increasing the order of the parabolic operator by two, consider the bi-harmonic equation in the same shrinking domain as in (3.1) with the zero Dirichlet boundary conditions on the lateral boundary $\partial Q_{0}$ :

$$
\left\{\begin{array}{c}
u_{t}=-u_{x x x x} \quad \text { in } Q_{0}=\{|x|<R(t),-1<t<0\}, \\
u=u_{x}=0 \quad \text { at } x= \pm R(t),-1 \leq t<0, \\
u(x, 0)=u_{0}(x) \quad \text { on }[-R(-1), R(-1)],
\end{array}\right.
$$

where $u_{0}(x)$ is a bounded and smooth function, $u_{0}( \pm R(-1))=0$.

First of all, obviously, in modern PDE theory, there are many very strong regularity results, proved for wide classes of linear and nonlinear equations including, of course, the simplest among others bi-harmonic one (4.1). We have mentioned some key results and papers before and will not indent to compete with those nice results and present no further references to this classic literature. The only issue we propose here is as follows: to show how to derive

a sharp boundary regularity criterion for (4.1) as a blow-up problem. 
As we have seen, these blow-up aspects of the regularity problem were already clearly revealed by Petrovskii in 1934. Though, for the second-order case, there are other equivalent approaches based on the positivity of the kernel and various order-preserving features, which fail for oscillatory kernels.

To be more precise, this blow-up study for (4.1) is performed for:

a generic class of solutions exhibiting a "centre subspace" behaviour.

In other words, as our Hermitian spectral theory of higher-order non self-adjoint linear operators in Sect. 5 shows, one can expect that, in addition to (4.3),

$\exists$ an infinite-dimensional set of "stable manifold" patterns,

for which the conditions of regularity of $(0,0)$ are all different.

It is principal for us that, to confirm the optimal character of the class of generic blow-up asymptotics, we will check (Sect. 7.7) that, by elementary spectral calculus,

the same asymptotic approach to the classic problem (3.1) by classic

Hermite spectral theory yields precisely Petrovskii's criterion in (3.11).

Note that the problem: (4.3) or (4.4), also exists for the heat equation (3.1) (cf. (3.14)), but here, by the Maximum Principle, positive solutions always belong to the generic class (4.3). For the bi-harmonic PDE (4.1), in a general setting, distinguishing generic patterns (4.3) from those in (4.4) is difficult and seems even impossible (or makes no sense).

\subsection{Slow growing factor $\varphi(\tau)$}

Thus, similar to (3.9), we need to assume that

$$
R(t)=(-t)^{\frac{1}{4}} \varphi(\tau), \quad \text { where } \tau=-\ln (-t) \rightarrow+\infty \quad \text { as } t \rightarrow 0^{-} .
$$

Here, the scaling main factor $(-t)^{1 / 4}$ naturally comes from the bi-harmonic kernel variables (see (4.11) and (5.3)), and $\varphi(\tau)>0$ is an unknown slow growing function satisfying ${ }^{12}$

$$
\varphi(\tau) \rightarrow+\infty, \quad \varphi^{\prime}(\tau) \rightarrow 0, \quad \text { and } \quad \frac{\varphi^{\prime}(\tau)}{\varphi(\tau)} \rightarrow 0 \quad \text { as } \tau \rightarrow+\infty .
$$

Moreover, as a sharper characterization of the above class of slow growing functions, we use the following criterion:

$$
\left(\frac{\varphi(\tau)}{\varphi^{\prime}(\tau)}\right)^{\prime} \rightarrow \infty \quad \text { as } \tau \rightarrow+\infty \quad\left(\varphi^{\prime}(\tau) \neq 0\right)
$$

This is a typical condition in blow-up analysis distinguishing classes of exponential (the limit is 0 ), power-like (a constant $\neq 0$ ), and slow-growing functions. See [79, pp. 390-400], where in Lemma 1 on p. 400, extra properties of slow-growing

\footnotetext{
${ }^{12}$ As we have mentioned, for the simpler case $\varphi(\tau), \varphi^{\prime}(\tau) \rightarrow 0$ as $\tau \rightarrow \infty$, the regularity was already proved by Mihailov in 1963 [67,68]; in a certain sense, this extended the Gevrey-like result (3.2) for $m=1$; see (3.3).
} 
functions (4.8) are proved. For instance, one can derive the following comparison of such $\varphi(\tau)$ with any power:

$$
\text { for any } \alpha>0, \quad \varphi(\tau) \ll \tau^{\alpha} \text { and } \varphi^{\prime}(\tau) \ll \tau^{\alpha-1} \text { for } \tau \gg 1 \text {. }
$$

Such estimates are useful in evaluating perturbation terms in the rescaled equations.

Thus, the monotone positive function $\varphi(\tau)$ in (4.6) is assumed to determine a sharp behaviour of the boundary of $Q_{0}$ near the shrinking point $(0,0)$ to guarantee its regularity. In Petrovskii's criterion (3.9), the almost optimal function, satisfying (4.7), (4.8), is

$$
\varphi_{1}(\tau) \sim 2 \sqrt{\ln \tau} \text { as } \tau \rightarrow+\infty,
$$

a dependence we have to compare our final results for the bi-harmonic equation with.

\subsection{First kernel scaling and layout}

By (4.6), we perform the similarity scaling

$$
u(x, t)=v(y, \tau), \quad \text { where } y=\frac{x}{(-t)^{1 / 4}} .
$$

Then the rescaled function $v(y, \tau)$ now solves the rescaled equation

$$
\left\{\begin{array}{l}
v_{\tau}=\mathbf{B}^{*} v \equiv-v_{y y y y}-\frac{1}{4} y v_{y} \quad \text { in } Q_{0}=\{|y|<\varphi(\tau), \tau>0\} \\
v=v_{y}=0 \text { at } y= \pm \varphi(\tau), \tau \geq 0 \\
v(0, y)=v_{0}(y) \equiv u_{0}(y) \quad \text { on }[-R(-1), R(-1)]
\end{array}\right.
$$

In view of the divergence (4.7), it follows that our final analysis will essentially depend on the spectral properties of the linear operator $\mathbf{B}^{*}$ on the whole line $\mathbb{R}$. We reflect this Hermitian spectral theory in the next Sect. 5, with application to the regularity criterion in Sect. 7. In particular, this differs our analysis from Kondrat'ev's classic one [43], where, as a rule, the rescaled boundary of the domain is key.

However, in Sect. 6, we begin the study of the regularity of the vertex $(0,0)$ of the bi-harmonic backward fundamental parabolae:

$$
R(t)=l(-t)^{\frac{1}{4}}, \quad \text { i.e., } \quad \varphi(\tau) \equiv l=\text { const. }>0 .
$$

Then the problem (4.12) is considered on the fixed interval $I_{l}=\{|y|<l\}$, so that the final conclusion entirely depends on spectral properties of $\mathbf{B}^{*}$ in $I_{l}$ with Dirichlet boundary conditions. Of course, then the spectral problem for $\mathbf{B}^{*}$ (not an operator pencil) becomes a particular case of the general setting developed in [43]. Nevertheless, since we need a sharp bound on the first eigenvalue, the clear conclusion on regilarity/irregularity becomes rather involved, where numerics are necessary to fix final details. In addition, as we pointed out, in more general setting for the fundamental backward paraboloids in $\mathbb{R}^{N}$, the existence, uniqueness, and regularity of solutions in Sobolev spaces was proved in a number of papers 
such as $[22,66-68]$, etc. Note that in [67, p. 45], the zero boundary data was understood in the mean sense (i.e., in the $L^{2}$-sense along a sequence of smooth internal contours "converging" to the boundary).

Thus, our conclusion is rather disappointing: unlike the classic heat equation (3.1), for the bi-harmonic equation (4.1), the vertexes of the fundamental parabolae (4.13) are not necessarily regular. ${ }^{13}$ For instance, on the basis of careful numerics, we show that:

$$
\begin{aligned}
& R(t)=4(-t)^{\frac{1}{4}} \Longrightarrow(0,0) \text { is regular, while } \\
& R(t)=5(-t)^{\frac{1}{4}} \Longrightarrow(0,0) \text { is irregular. }
\end{aligned}
$$

Moreover, in the latter case, the vertex $(0,0)$ is singular, i.e.,

$$
\text { in general, } u(x, t) \text { is unbounded as }(x, t) \rightarrow\left(0,0^{-}\right) \text {. }
$$

We also claim that, by a continuity argument, there exists some $l_{1} \in(4,5)$ such that $(0,0)$ is irregular, but not singular, i.e., a finite limit $u\left(0,0^{-}\right)$exists.

These regularity variations for constant functions $\varphi(\tau) \equiv l$ inevitably impose a special restriction for the study in Sect. 7 of unbounded $\varphi(\tau)$ 's satisfying (4.7), where we derive a Petrovskii-like "criterion" of regularity. Namely, the irregularity condition is obtained in terms of an Osgood-Dini-type integral condition, while, in an accordance with (4.14), for the regularity, a special "oscillatory cut-off" of the lateral boundary must be applied.

In Sect. 8, we discuss the extensions of the boundary regularity analysis to $2 m$ th-order poly-harmonic operators

$$
u_{t}=(-1)^{m+1} D_{x}^{2 m} u \quad \text { in } Q_{0} \quad(m \geq 2),
$$

with the zero Dirichlet conditions, as well as for the same $N$-dimensional problem for (1.4) in $Q_{0}$, where the shape of the shrinking domain $Q_{0}$ is also of importance for the boundary regularity. For instance, one can consider the radial $Q_{0}$ given in (4.1), where $|x|$ stands for the radial spatial variable. We also discuss regularity conditions for the third-order linear dispersion equations, for a quasilinear fourthorder porous-medium-type equation (the PME-4), and for the linear wave (beam) equation of the fourth order.

The present paper aims to give a first insight into principal difficulties of a sharp study of boundary regularity for higher-order parabolic and other evolution PDEs. Such a study inevitably generates a number of difficult mathematical problems, which, partially, do not appear in the second-order case, or can be avoided by positivity kernel properties. We must admit that some of them are not and even cannot be completely rigorously solved. The main problem of concern is that distinguishing generic patterns in (4.3) from non-generic ones in (4.4) is not possible

\footnotetext{
${ }^{13}$ It is worth comparing this with the following well-known (since 1986) negative conclusion in elliptic theory: for fourth-order elliptic equations in dimensions $N \geq 8$, the vertex of a cone can be irregular-singular, i.e., solutions are unbounded at the vertex, [64]. For $5 \leq N \leq 7$, the biharmonic capacity settles the regularity result in Wiener's sense [60], while $N<4=2 m$ is done by Sobolev embeddings.
} 
in general. Here, delicate boundary layer theory is essentially involved, where we ought to use some accurate numerical calculations by the MatLab whenever necessary to avoid huge technical digressions (using those seems also inevitable for truly $2 m$ th-order parabolic equations with large enough $m \geq 2$ ). Regardless a certain rigorous incompleteness of the mathematical analysis in some steps (which seems to be inevitable in general), we decide to demonstrate the whole machinery of the asymptotic blow-up methods in parabolic boundary regularity theory, and hope that this will help to attract some attentive Readers to improve the results when necessary. It is worth mentioning that, as can be seen in many directions of modern PDE theory of linear and nonlinear equations, the transition to higher-order models is accompanying by a dramatic increase of the complexity of the methods involved to achieve in a rigorous manner the necessary (basic or not) results. This increase of complexity sometimes is measured in orders, and often desired rigorous proofs can be illusive in a sufficient generality. A suitable restriction of the generality to achieve rigorous conclusions, though being an important research step, can lead to an extended and often unjustified number of artificial hypothesis and even yield non-constructive assumptions (i.e., those that cannot be checked in a reasonable finite time or at all). In what follows, we prefer to avoid stating such theorems with non-constructive hypothesis, though this is indeed plausible in a few places. ${ }^{14}$ In this case, we believe that the exchange of the ideas and methods, even in the case of a certain lack of a completely rigorous justification, can be key for further improvements and developing more consistent mathematical PDE theory. Of course, this imposes certain restrictions on the paper style, but the author hopes that the interested Reader will easily distinguish the rigorous and non-rigorous arguments to be used.

\section{Fundamental solution and Hermitian spectral theory for $\left\{\mathrm{B}, \mathrm{B}^{*}\right\}$}

For convenience of the further study, we consider the $2 m$ th-order poly-harmonic equation (4.16), and will describe the necessary spectral properties of the linear differential operator, which is the analogy of that in (4.12) for any $m \geq 2$,

$$
\mathbf{B}^{*}=(-1)^{m+1} D_{y}^{2 m}-\frac{1}{2 m} y D_{y}
$$

and of its adjoint $\mathbf{B}$ in the standard $L^{2}$-metric given by

$$
\mathbf{B}=(-1)^{m+1} D_{y}^{2 m}+\frac{1}{2 m} y D_{y}+\frac{1}{2 m} I \quad(I \text { denotes the identity }) .
$$

Both operators are not symmetric for any $m \geq 2$ and do not admit a self-adjoint extension. We will follow [15] in presenting necessary spectral theory.

\footnotetext{
14 "The main goal of a mathematician is not proving a theorem, but an effective investigation of the problem...", A.N. Kolmogorov, 1980s (the author apologizes for a non-literal translation from the Russian).
} 


\subsection{The fundamental solution and its sharp estimates}

We begin with determining the spectrum and the eigenfunctions of the adjoint operator B, which appears in constructing the fundamental solution of (4.16) that takes the standard self-similar form

$$
b(x, t)=t^{-\frac{1}{2 m}} F(y), \quad y=\frac{x}{t^{1 / 2 m}} .
$$

Substituting $b(x, t)$ into (4.16) yields that the radially symmetric profile $F(y)$ is the unique even integrable solution of the linear ODE

$$
\mathbf{B} F=0 \quad \text { in } \quad \mathbb{R}, \quad \int_{\mathbb{R}} F=1,
$$

so it is a null eigenfunction of $\mathbf{B}$. Taking the Fourier transform leads to

$$
F(y)=\alpha_{0} \int_{0}^{\infty} \mathrm{e}^{-s^{2 m}} \cos (s y) \mathrm{d} s
$$

where $\alpha_{0}>0$ is the normalization constant, and, more precisely [17],

$$
F(y)=\frac{1}{\sqrt{2 \pi}} \int_{0}^{\infty} \mathrm{e}^{-s^{2 m}} \sqrt{s|y|} J_{-\frac{1}{2}}(s|y|) \mathrm{d} s \text { in } \mathbb{R},
$$

where $J_{\nu}$ denotes Bessel's function. The rescaled kernel $F(y)$ then satisfies a standard pointwise exponential estimate [16]

$$
|F(y)| \leq D \mathrm{e}^{-d_{0}|y|^{\alpha}} \quad \text { in } \mathbb{R}, \quad \text { where } \alpha=\frac{2 m}{2 m-1}
$$

and $D$ and $d_{0}$ are some positive constants ( $d_{0}$ to be specified below). Such optimal exponential estimates of the fundamental solutions of higher-order parabolic equations are well-known and were first obtained by Evgrafov-Postnikov (1970) and Tintarev (1982); see Barbatis [7,8] for key references and results.

However, as a crucial issue for the further boundary point regularity study, we will need a sharper, than given by (5.7), asymptotic behaviour of the rescaled kernel $F(y)$ as $y \rightarrow+\infty$. To get that, we re-write the Eq. (5.4) on integration once as

$$
(-1)^{m+1} F^{(2 m-1)}+\frac{1}{2 m} y F=0 \text { in } \mathbb{R} .
$$

Using standard classic WKBJ asymptotics, we substitute into (5.8) the function

$$
F(y) \sim y^{-\delta_{0}} \mathrm{e}^{a y^{\alpha}} \quad \text { as } y \rightarrow+\infty,
$$

exhibiting two scales. This gives the algebraic equation for $a$,

$$
(-1)^{m}(\alpha a)^{2 m-1}=\frac{1}{2 m}, \quad \text { and } \quad \delta_{0}=\frac{m-1}{2 m-1}>0 .
$$

Note that the slow algebraically decaying factor $y^{-\delta_{0}}$ in (5.9) is available for any $m \geq 2$ and is absent for $m=1$ for the pure exponential positive Gaussian profile

$$
F(y)=\frac{1}{2 \sqrt{\pi}} \mathrm{e}^{-|y|^{2} / 4} \quad(m=1) .
$$


By construction, one needs to get the root $a$ of (5.10) with the maximal Re $a<0$. This yields (see e.g., [7,8] and [30, p. 141])

$$
\begin{aligned}
a & =\frac{2 m-1}{(2 m)^{\alpha}}\left[-\sin \left(\frac{\pi}{2(2 m-1)}\right)+\mathrm{i} \cos \left(\frac{\pi}{2(2 m-1)}\right)\right] \\
& \equiv-d_{0}+\mathrm{i} b_{0} \quad\left(d_{0}>0\right)>0 .
\end{aligned}
$$

Finally, this gives the following double-scale asymptotic of the kernel:

$$
F(y)=y^{-\delta_{0}} \mathrm{e}^{-d_{0} y^{\alpha}}\left[C_{1} \sin \left(b_{0} y^{\alpha}\right)+C_{2} \cos \left(b_{0} y^{\alpha}\right)\right]+\cdots \quad \text { as } y \rightarrow+\infty,
$$

where $C_{1,2}$ are real constants, $\left|C_{1}\right|+\left|C_{2}\right| \neq 0$. In (5.13), we present the first two leading terms from the $m$-dimensional bundle of exponentially decaying asymptotics.

In particular, for the bi-harmonic equation (4.1), we have

$$
m=2: \quad \alpha=\frac{4}{3}, \quad d_{0}=3 \times 2^{-\frac{11}{3}}, \quad b_{0}=3^{\frac{3}{2}} \times 2^{-\frac{11}{3}}, \quad \text { and } \quad \delta_{0}=\frac{1}{3} .
$$

\subsection{The discrete real spectrum and eigenfunctions of $B$}

We describe the spectrum $\sigma(\mathbf{B})$ of $\mathbf{B}$ in the space $L_{\rho}^{2}(\mathbf{R})$ with the exponential weight

$$
\rho(y)=\mathrm{e}^{a|y|^{\alpha}}>0 \quad \text { in } \mathbb{R} \quad\left(\alpha=\frac{2 m}{2 m-1}\right),
$$

where $a \leq 2 d_{0}$ is any fixed positive constant. We next introduce a standard Hilbert (a weighted Sobolev) space of functions $H_{\rho}^{2 m}(\mathbb{R})$ with the inner product and the norm

$$
\langle v, w\rangle_{\rho}=\int_{\mathbb{R}} \rho(y) \sum_{k=0}^{2 m} D_{y}^{k} v(y) \overline{D_{y}^{k} w(y)} \mathrm{d} y, \quad\|v\|_{\rho}^{2}=\int_{\mathbb{R}} \rho(y) \sum_{k=0}^{2 m}\left|D_{y}^{k} v(y)\right|^{2} \mathrm{~d} y .
$$

Then $H_{\rho}^{2 m}(\mathbb{R}) \subset L_{\rho}^{2}(\mathbb{R}) \subset L^{2}(\mathbb{R})$, and $\mathbf{B}$ is a bounded linear operator from $H_{\rho}^{2 m}(\mathbb{R})$ to $L_{\rho}^{2}(\mathbb{R})$. With these definitions, the spectral properties of the operator $\mathbf{B}$ are given by:

\section{Lemma 5.1.}

(i) The spectrum of $\mathbf{B}$ comprises real simple eigenvalues only,

$$
\sigma(\mathbf{B})=\left\{\lambda_{k}=-\frac{k}{2 m}, k=0,1,2, \ldots\right\} .
$$

(ii) The eigenfunctions $\psi_{k}(y)$ are given by

$$
\psi_{k}(y)=\frac{(-1)^{k}}{\sqrt{k !}} D_{y}^{k} F(y)
$$

and form a complete set in $L^{2}(\mathbb{R})$ and in $L_{\rho}^{2}(\mathbb{R})$.

(iii) The resolvent $(\mathbf{B}-\lambda I)^{-1}$ for $\lambda \notin \sigma(\mathbf{B})$ is a compact integral operator in $L_{\rho}^{2}(\mathbb{R})$. 
The operators $\mathbf{B}$ have zero Morse index, i.e., no eigenvalues have positive real part. Note that in the classic Hermite case $m=1$ (in fact, the only self-adjoint case), the spectrum is again given by (5.16) and the eigenfunction formula (5.17) with the rescaled kernel (5.11) generates Hermite polynomials; see [10, p. 48] for a full spectral account for the operator $\mathbf{B}$ in $\mathbb{R}^{N}$.

\subsection{The polynomial eigenfunctions of the operator $B^{*}$}

We now consider the operator $(5.1)$ in the weighted space $L_{\rho^{*}}^{2}(\mathbb{R})\left(\langle\cdot, \cdot\rangle_{\rho^{*}}\right.$ and $\|\cdot\|_{\rho^{*}}$ are the inner product and the norm) with the "adjoint" exponentially decaying weight function

$$
\rho^{*}(y) \equiv \frac{1}{\rho(y)}=\mathrm{e}^{-a|y|^{\alpha}}>0 .
$$

We ascribe to $\mathbf{B}^{*}$ the domain $H_{\rho^{*}}^{2 m}(\mathbb{R})$, which is dense in $L_{\rho^{*}}^{2}(\mathbb{R})$, and then

$$
\mathbf{B}^{*}: H_{\rho^{*}}^{2 m}(\mathbb{R}) \rightarrow L_{\rho^{*}}^{2}(\mathbb{R})
$$

is a bounded linear operator. $\mathbf{B}$ is adjoint to $\mathbf{B}^{*}$ in the usual sense: denoting by $\langle\cdot, \cdot\rangle$ the inner product in the dual space $L^{2}(\mathbb{R})$, we have

$$
\langle\mathbf{B} v, w\rangle=\left\langle v, \mathbf{B}^{*} w\right\rangle \quad \text { for any } v \in H_{\rho}^{2 m}(\mathbb{R}), w \in H_{\rho^{*}}^{2 m}(\mathbb{R}) .
$$

The eigenfunctions of $\mathbf{B}^{*}$ take a particularly simple polynomial form and are as follows:

\section{Lemma 5.2.}

(i) $\sigma\left(\mathbf{B}^{*}\right)=\sigma(\mathbf{B})$.

(ii) The eigenfunctions $\psi_{k}^{*}(y)$ of $\mathbf{B}^{*}$ are polynomials in $y$ of order $k$ given by

$$
\psi_{k}^{*}(y)=\frac{1}{\sqrt{k !}} \sum_{j=0}^{[k / 2 m]} \frac{(-1)^{m j}}{j !} D_{y}^{2 m j} y^{k}, \quad k=0,1,2, \ldots,
$$

and form a complete subset in $L_{\rho^{*}}^{2}(\mathbb{R})$.

(iii) $\mathbf{B}^{*}$ has compact resolvent $\left(\mathbf{B}^{*}-\lambda I\right)^{-1}$ in $L_{\rho^{*}}^{2}(\mathbb{R})$ for $\lambda \notin \sigma\left(\mathbf{B}^{*}\right)$.

Of course, for $m=1$, (5.20) yields standard Hermite polynomials, so, for $m \geq 2$, we call (5.20) generalized Hermite polynomials. With the definition (5.20) of the adjoint basis, by integrating by parts, the orthonormality condition holds $\left(\delta_{k l}\right.$ is the Kronecker delta):

$$
\left\langle\psi_{k}, \psi_{l}^{*}\right\rangle=\delta_{k l} \text { for any } k, l \geq 0 .
$$

For $m=2$ (this case will be treated in greater detail), the first eigenfunctions are

$$
\begin{aligned}
& \psi_{0}(y)=1, \quad \psi_{1}(y)=y, \quad \psi_{2}(y)=\frac{1}{\sqrt{2}} y^{2}, \quad \psi_{3}(y)=\frac{1}{\sqrt{6}} y^{3}, \\
& \psi_{4}(y)=\frac{1}{\sqrt{24}}\left(y^{4}+24\right), \quad \psi_{5}(y)=\frac{1}{2 \sqrt{30}}\left(y^{5}+120 y\right), \psi_{6}(y)=\frac{1}{\sqrt{6 !}}\left(y^{6}+360 y^{2}\right),
\end{aligned}
$$


etc., with the corresponding eigenvalues $0,-\frac{1}{4},-\frac{1}{2},-\frac{3}{4},-1,-\frac{5}{4},-\frac{3}{2}$.

\section{The vertex of fundamental parabolae can be regular or irregular}

Thus, consider the backward fundamental parabolae given by (4.13). We continue denote by $\mathbf{B}^{*}$ the corresponding linear operator

$$
\mathbf{B}^{*} v=-v^{(4)}-\frac{1}{4} y v^{\prime} \quad \text { in } I_{l}, \quad v=v^{\prime}=0 \quad \text { at } y= \pm l,
$$

with the standard definition of the domain, etc. Here, $\mathbf{B}^{*}$ is a regular ordinary differential operator with bounded smooth coefficients and a discrete spectrum $\sigma\left(\mathbf{B}^{*}\right)[71]$. Note that $\mathbf{B}^{*}$ is not symmetric and does not admit a self-adjoint extension, so that $\sigma\left(\mathbf{B}^{*}\right)$ is not necessarily real. However, we will observe a lot of real eigenvalues to be explained by a branching phenomenon with a clear shooting nature. By $\lambda_{k}(l)$, we denote $l$-branches of eigenvalues of $\mathbf{B}^{*}$ depending on the length $l>0$. In view of Sect. 5 , the limit $l \rightarrow+\infty$ is of the particular interest.

The regularity criterion of the vertex is now easy:

vertex $(0,0)$ of parabolae (4.13) is regular iff $\sigma\left(\mathbf{B}^{*}\right) \subset \mathbb{C}_{-}=\mathbb{C} \cap\{\operatorname{Re} \lambda<0\}$.

Then the solution $v(y, \tau)$ (and any $u(x, t))$ gets exponentially small: as $\tau \rightarrow+\infty$,

$$
v(y, \tau) \sim \mathrm{e}^{\lambda_{0} \tau} \rightarrow 0 \Longrightarrow|u(x, t)| \sim O\left(t^{-\operatorname{Re} \lambda_{0}}\right) \rightarrow 0 \quad \text { as } t \rightarrow 0^{-},
$$

where $\lambda_{0}(l)$ is the eigenvalue of $\mathbf{B}^{*}$ with the maximal negative Re (in fact, $\lambda_{0}(l)$ turns out to be always real). Let us now briefly describe main steps of the current study.

\subsection{The vertex $(0,0)$ is regular for not that large $l<3.9779 \ldots$}

This conclusion is simple: as a standard practice, by multiplying in $L^{2}\left(I_{l}\right)$ the eigenfunction equation

$$
\mathbf{B}^{*} \Psi^{*} \equiv-\left(\Psi^{*}\right)^{(4)}-\frac{1}{4} y\left(\Psi^{*}\right)^{\prime}=\lambda \Psi^{*}
$$

by the complex conjugate $\bar{\Psi}^{*}$ and summing up with the result of multiplying the complex conjugate equation by $\Psi^{*}$, on integration by parts, one obtains

$$
(\lambda+\bar{\lambda}) \int\left|\Psi^{*}\right|^{2}=-2 \int\left|\Psi^{\prime \prime}\right|^{2}+\frac{1}{4} \int\left|\Psi^{*}\right|^{2} .
$$

Using the Poincaré inequality:

$$
\int\left|\Psi^{\prime \prime}\right|^{2} \geq \Lambda_{0}(l) \int|\Psi|^{2}, \quad \Psi \in H_{0}^{2}\left(I_{l}\right) ; \quad \Lambda_{0}(l)=\frac{1}{l^{4}} \Lambda_{0}(1), \quad \Lambda_{0}(1)=31.3 . .(6,6)
$$

where $\Lambda_{0}(1)$ is the first eigenvalue of $D_{y}^{(4)}>0$ in $H_{0}^{2}\left(I_{1}\right)$, we obtain from (6.5):

$$
(\lambda+\bar{\lambda}) \int\left|\Psi^{*}\right|^{2} \leq-\left[\frac{2 \Lambda_{0}(1)}{l^{4}}-\frac{1}{4}\right] \int\left|\Psi^{*}\right|^{2} .
$$


Therefore, the regularity of the vertex $(0,0)$ is guaranteed for (this is not sharp but close):

$$
\frac{2 \Lambda_{0}(1)}{l^{4}}-\frac{1}{4}>0 \Longrightarrow l<l_{*}=\left[8 \Lambda_{0}(1)\right]^{\frac{1}{4}}=3.9779 \ldots
$$

\subsection{First eigenvalues of $B^{*}$ are real}

Here and later on in a few special technically difficult cases, we are going to rely on a clear numerical evidence for our conclusions. To this end, we use the MatLab solver bvp4c with the enhanced accuracy and tolerances

$$
\text { from } 10^{-10} \text { to the minimal admitted } 10^{-13} \text {. }
$$

Here we use a two-parameter shooting over real $\lambda$ 's and l's. This will guarantee that real eigenvalues, which can be very small, are computed correctly.

In Fig. 1, we demonstrate first eigenfunctions of the operator $\mathbf{B}^{*}$ in (6.1) for $l=1$, where $\Psi_{0}^{*}, \Psi_{2}^{*}$, and $\Psi_{4}^{*}$ are even functions, while $\Psi_{1}^{*}$ and $\Psi_{3}^{*}$ are odd ones. Note that, by obvious reasons, the first eigenvalue

$$
\lambda_{0}(1)=-31.16 \ldots
$$

is rather close to the eigenvalue $-\Lambda_{0}(1)=-31.3 \ldots$ in $(6.6)$ of the self-adjoint counterpart $-D_{y}^{(4)}$, i.e., the non-symmetric perturbation $-\frac{1}{4} y D_{y}$ is negligible. Actually, the "Sturmian geometric structure" of eigenfunctions in Fig. 1: each $\Psi_{k}^{*}(y)$ has precisely $k$ zeros in $I_{1}$, which is true for $-D_{y}^{(4)}$ [19], clearly remains

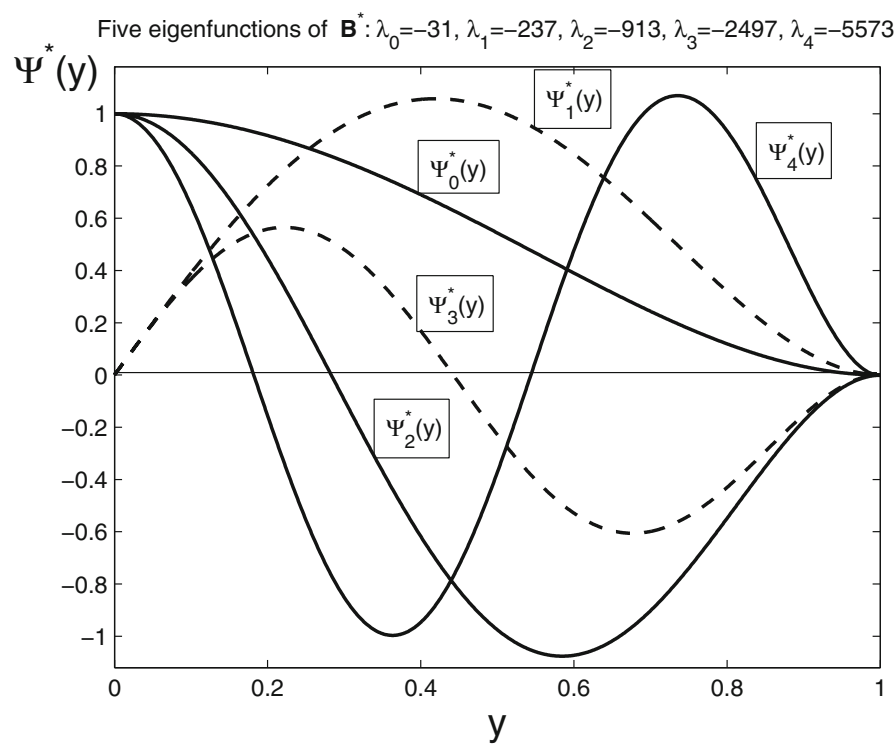

FiguRE 1. Five first real eigenfunctions of the operator (6.1) for $l=1$ 


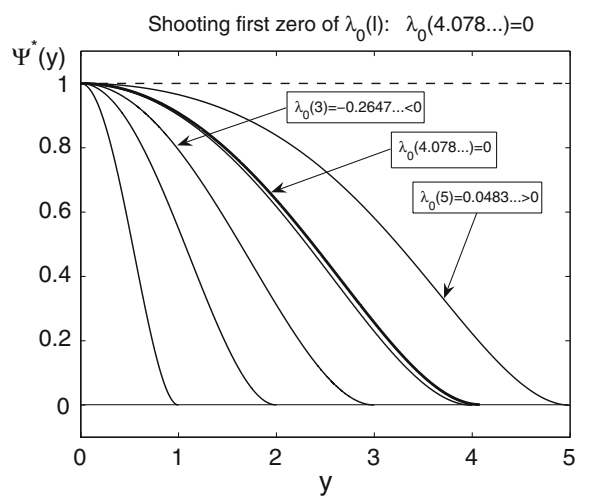

(a) first zero $l_{1}=4.08 \ldots$

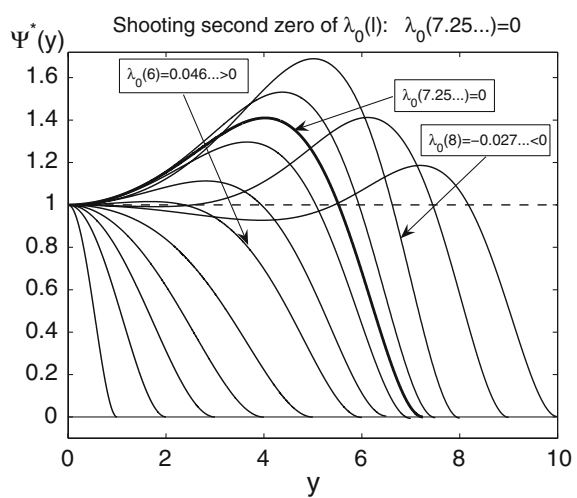

(b) second zero $l_{2}=7.25 \ldots$

Figure 2. Shooting zeros of $\lambda_{0}(l)$ via bvp4c: first zero (a) and the second one $(\mathbf{b})$

valid for $\mathbf{B}^{*}$. Moreover, we have observed that the first eigenfunctions of $\mathbf{B}^{*}$ and $-D_{y}^{(4)}$ for $l=1$ coincide within the accuracy $0.5 \times 10^{-2}$. In fact, this suggests to get $\lambda_{k}(l)$ by branching at $\mu=0^{+}$from eigenfunctions of $-D_{y}^{(4)}<0$ by using the following family for $\mu \in\left[0, \frac{1}{4}\right]$ (a homotopy path) of operators in $H_{0}^{4}\left(I_{l}\right)$ :

$$
\mathbf{B}_{\mu}^{*}=-D_{y}^{(4)}-\mu y D_{y}^{\prime}=\mathbf{B}^{*} \text { for } \mu=\frac{1}{4} .
$$

For $\mu=0$, the self-adjoint $\mathbf{B}_{0}^{*}=-D_{y}^{(4)}$ has the desired real spectrum and complete-closed set of eigenfunctions; see [36] and [83] for classic perturbation/branching theory. Later on, we present another branching explanation of the origin of real eigenvalues/eigenfunctions of the non self-adjoint operator $\mathbf{B}^{*}$ by using results from Sect. 5 reflected $l=\infty$.

\section{3. $\lambda_{0}(l)$ Changes sign: numerical evidence}

It is clear that, for large $l$, the non-symmetric perturbation $-\frac{1}{4} y D_{y}$ becomes essential in $\mathbf{B}^{*}$, and Sturm's zero property in Fig. 1 does not remain true. In Fig. 2(a,b), we present the shooting results of numerical calculating via the bvp4c solver (a 2D shooting as above) of first three roots of the branch of the first eigenvalue $\left\{\lambda_{0}(l)\right\}$ :

$$
\lambda_{0}(l)=0 \text { at first three roots } l_{1}=4.077 \ldots, l_{2}=7.287 \ldots, \text { and } l_{3}=12.644 \ldots .
$$

Note that $l_{1}$ is about $3 \%$ close to the bound $l_{*}=3.9779 \ldots$ derived in $(6.8)$. In Table 1, we present other related numerical results for $l$ around two roots $l_{1}$ and $l_{2}$. 
TABLE 1 . Some values of $\lambda_{0}(l)$

\begin{tabular}{lc}
\hline$l$ & $\lambda_{0}(l)$ \\
\hline 1 & $-31.16 \ldots$ \\
2 & $-1.83 \ldots$ \\
3 & $-0.2647 \ldots$ \\
4 & $-0.008152 \ldots$ \\
4.075 & $-0.000236 \ldots$ \\
$4.0775 \approx l_{1}$ & $0.0000113 \ldots$ \\
4.08 & $0.00026 \ldots$ \\
4.1 & $0.0022 \ldots$ \\
4.2 & $0.011 \ldots$ \\
5 & $0.0483 \ldots$ \\
5 & $0.0483 \ldots$ \\
6 & $0.046 \ldots$ \\
$7.25 \sim l_{2}$ & $0.00167 \ldots$ \\
7.5 & $-0.0097 \ldots$ \\
8 & $-0.027 \ldots$ \\
9 & $-0.018 \ldots$ \\
\hline
\end{tabular}

In Fig. 3, we show the enhanced results of a one-parameter shooting of the eigenfunctions $\Psi_{0}^{*}(y)$ via the ode45 solver, where we put $\lambda=0$ and the only shooting parameter is the value of the second-order derivative $\left(\Psi^{*}\right)^{\prime \prime}(0)$, with $\Psi^{*}(0)=1$ and $\left(\Psi^{*}\right)^{\prime}(0)=\left(\Psi^{*}\right)^{\prime \prime \prime}(0)=0$ by symmetry. Thus, we obtain the following sharp estimates of the first four roots of $\lambda_{0}(l)$ :

$$
\lambda_{0}\left(l_{4}\right)=0: l_{1}=4.077 \ldots, l_{2}=7.287 \ldots, l_{3}=12.6444 \ldots, l_{4}=17.318 \ldots
$$

This Figure clearly shows a general idea how to prove that $\mathbf{B}^{*}$ in (6.1) has infinitely many real eigenvalues: in view of the oscillatory and exponentially growing behaviour of generic solutions as $y \rightarrow+\infty$, the eigenvalue problem with two real parameters $\lambda$ and $\left(\Psi^{*}\right)^{\prime \prime}(0)$ (for symmetric functions) is well posed to satisfy also two Dirichlet boundary conditions posed at $y=l$. Since the problem is regular, with such growth of eigenvalues (characteristic values of $\mathbf{B}^{*} \mathbf{B}$ ), the eigenfunction subset is expected to be complete. If it is also closed in $L^{2}\left(I_{l}\right)$, no other complex eigenvalues are possible. These require further analysis, which is not key now.

Thus, taking into account these numerical results, in view of (6.2), we conclude that

$(0,0)$ is: regular for $l \in\left(0, l_{1}\right)$, irregular for $l \in\left[l_{1}, l_{2}\right]$, regular for $l \in\left(l_{2}, l_{3}\right)$,

etc. In addition, since $\lambda_{0}\left(l_{1,2}\right)=0$, one can expect that for $l=l_{1,2}$, the vertex $(0,0)$ remains irregular, but now it is not singular, i.e., under a natural nonorthogonality condition, $u\left(0,0^{-}\right) \neq 0$ is finite. Same happens for other roots $\lambda_{k}$ 


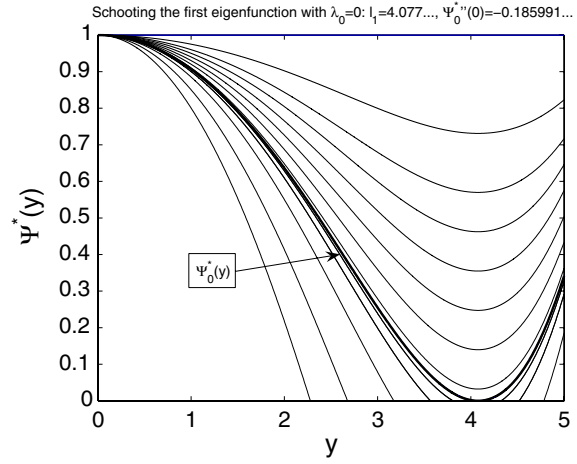

(a) first zero, $\left(\Psi^{*}\right)^{\prime \prime}(0)=-0.185991 \ldots$

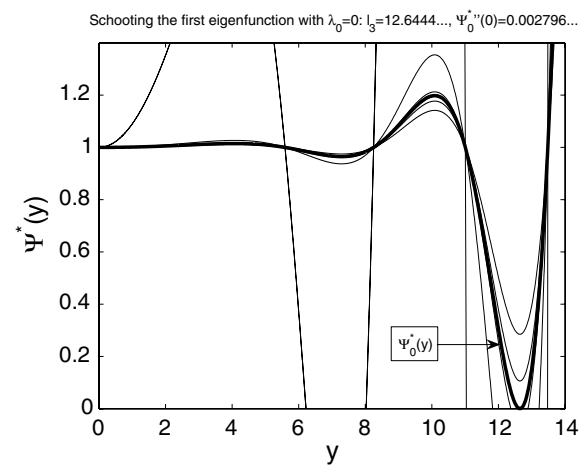

(c) third zero, $\left(\Psi^{*}\right)^{\prime \prime}(0)=0.002796 \ldots$

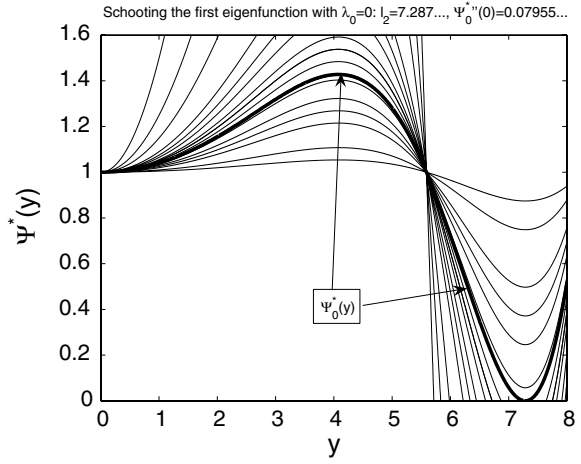

(b) second zero, $\left(\Psi^{*}\right)^{\prime \prime}(0)=0.07955 \ldots$

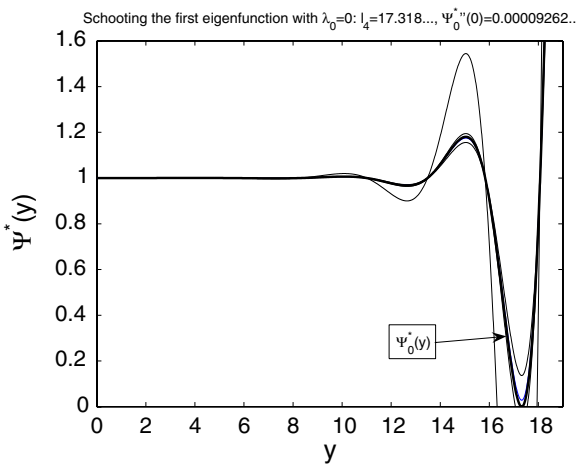

(d) fourth zero, $\left(\Psi^{*}\right)^{\prime \prime}(0)=0.00009262 \ldots$

Figure 3. Shooting eigenfunctions $\Psi_{0}^{*}(y)$ for $\lambda_{0}=0$ via the ode45: $l_{1}=4.077 \ldots$ (a), $l_{2}=7.287 \ldots$ (b), $l_{3}=12.6444 \ldots$ (c), and $l_{4}=17.318 \ldots(\mathbf{d})$

of $\lambda_{0}(l)$, provided that the rest of eigenvalues are "ordered" (i.e., the real $\lambda_{0}(l)$ remains the leading one). This has not been proved for arbitrary $l>0$, but is indeed true for $l \gg 1$, by an asymptotic treatment.

\subsection{Boundary layer and branching at $l=+\infty$}

The phenomenon of the boundary layer (BL) occurring as $l \rightarrow \infty$ is explained by Fig. 4. It follows that already at $l=20$, the first eigenfunction $\Psi_{0}^{*}(y)$ of $\mathbf{B}^{*}$ has a matching structure of the Boundary Layer for $y \approx l$ with the constant eigenfunction $\psi_{0}^{*}(y) \equiv 1$ (see (5.20)), which dominates for $l \leq 12$.

The BL structure here is simple and standard; see more details in Sect. 7.2, where the BL is constructed for the non-stationary parabolic PDE. Briefly, asymptotically sharp, in the BL for $y \sim l \gg 1$, Eq. (6.4) for $\left|\lambda_{0}(l)\right| \ll 1$ being asymptotically small (see (6.20)) reads 


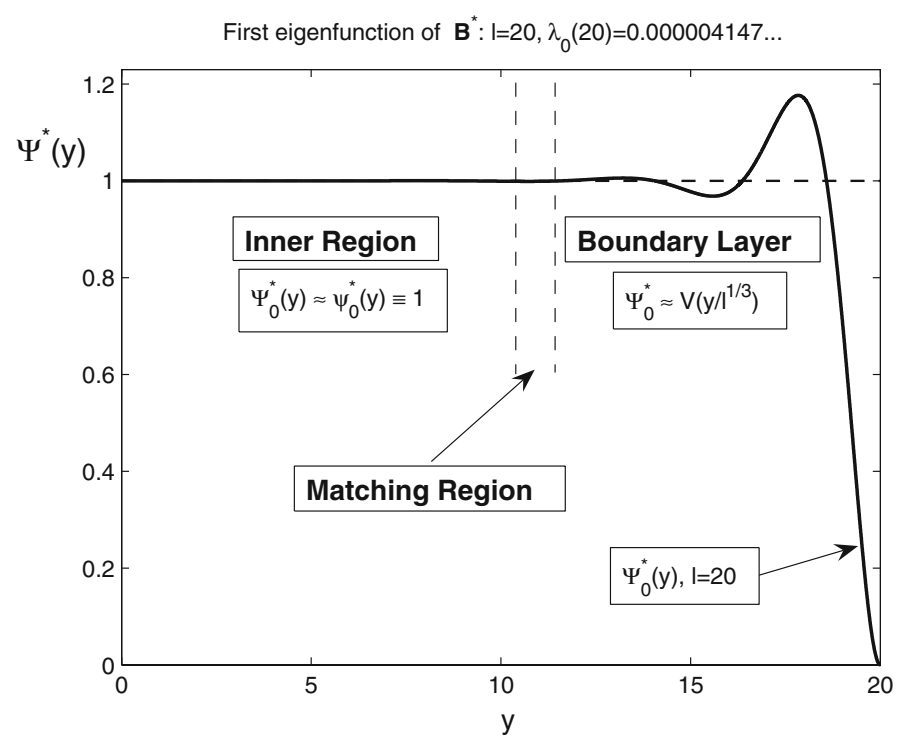

FiguRE 4. A typical boundary layer structure for $\Psi_{0}^{*}(y), l=20$

$$
\begin{aligned}
& -\left(\Psi^{*}\right)^{(4)}-\frac{1}{4} l\left(\Psi^{*}\right)^{\prime}+\cdots=0 \Longrightarrow \Psi^{*}=V(z)+\cdots, \quad z=l^{\frac{1}{3}} y, \quad \text { where } \\
& -V^{(4)}-\frac{1}{4} V^{\prime}=0, \quad z \in\left(0, l^{\frac{4}{3}}\right) ; \quad V=V^{\prime}=0, z=l^{\frac{4}{3}} ; \quad V(0)=1 .
\end{aligned}
$$

The BL-function $V$ is given explicitly (cf. (7.10)): denoting $b=2^{-5 / 3}$ and $a=$ $\sqrt{3} b$

$$
\begin{gathered}
V(z)=C_{3}-\mathrm{e}^{-b z}\left[C_{1} \cos (a z)+C_{2} \sin (a z)\right], \quad C_{1}=\frac{\mathrm{e}^{b l^{4 / 3}}\left[\cos \left(a l^{4 / 3}\right)-\frac{1}{\sqrt{3}} \sin \left(a l^{4 / 3}\right)\right]}{1-\mathrm{e}^{b l^{4 / 3}\left[\cos \left(a l^{4 / 3}\right)-\frac{1}{\sqrt{3}} \sin \left(a l^{4 / 3}\right)\right]},} \\
C_{2}=\frac{\mathrm{e}^{b l^{4 / 3}}\left[\sin \left(a l^{4 / 3}\right)+\frac{1}{\sqrt{3}} \cos \left(a l^{4 / 3}\right)\right]}{1-\mathrm{e}^{b l^{4 / 3}}\left[\cos \left(a l^{4 / 3}\right)-\frac{1}{\sqrt{3}} \sin \left(a l^{4 / 3}\right)\right]}, \quad C_{3}=\frac{1}{1-\mathrm{e}^{b l^{4 / 3}}\left[\cos \left(a l^{4 / 3}\right)-\frac{1}{\sqrt{3}} \sin \left(a l^{4 / 3}\right)\right]} .
\end{gathered}
$$

The eigenvalue $\lambda_{0}(l)$ for $l \gg 1$ is then obtained by matching in $\mathbb{R}$ : extending $\hat{\Psi}_{0}^{*}=\Psi_{0}^{*} H(l-y)$, where $H$ is the Heaviside function, we have (see Sect. 7.3 for details)

$$
\begin{aligned}
& \left(\hat{\Psi}_{0}^{*}\right)^{(4)}(y)=\left(\Psi_{0}^{*}\right)^{(4)}(y) H-\left(\Psi_{0}^{*}\right)^{\prime \prime \prime}(l) \delta(y-l)-\left(\Psi_{0}^{*}\right)^{\prime \prime}(l) \delta^{\prime}(y-l), \\
& \quad \text { where }\left(\Psi_{0}^{*}\right)^{\prime \prime}(l)=l^{\frac{2}{3}} V^{\prime \prime}\left(l^{\frac{4}{3}}\right)+\cdots, \quad\left(\Psi_{0}^{*}\right)^{\prime \prime \prime}(l)=l V^{\prime \prime \prime}\left(l^{\frac{4}{3}}\right)+\cdots .
\end{aligned}
$$


Then (6.4) for $\lambda_{0}(l)$ reads

$$
\mathbf{B}^{*} \hat{\Psi}^{*}-l V^{\prime \prime \prime}\left(l^{\frac{4}{3}}\right) \delta(y-l)-l^{\frac{2}{3}} V^{\prime \prime}\left(l^{\frac{4}{3}}\right) \delta^{\prime}(y-l)+\cdots=\lambda_{0}(l) \hat{\Psi}_{0}^{*} \quad \text { in } \mathbb{R} .
$$

Finally, substituting $\hat{\Psi}_{0}^{*}=\psi_{0}^{*}+w \equiv 1+w$, where $w \perp \psi_{0}$, yields

$$
\mathbf{B}^{*} w-l V^{\prime \prime \prime}\left(l^{\frac{4}{3}}\right) \delta(y-l)-l^{\frac{2}{3}} V^{\prime \prime}\left(l^{\frac{4}{3}}\right) \delta^{\prime}(y-l)+\cdots=\lambda_{0}(l)+\cdots .
$$

Hence, by the orthogonality condition to $\psi_{0}(y)=F(y)$ (recall that $\mathbf{B}^{*} w \perp \psi_{0}$ ), (6.18) implies the following asymptotic expression for the eigenvalue:

$$
\begin{aligned}
\lambda_{0}(l) & =-\left\langle l V^{\prime \prime \prime}\left(l^{\frac{4}{3}}\right) \delta(y-l)-l^{\frac{2}{3}} V^{\prime \prime}\left(l^{\frac{4}{3}}\right) \delta^{\prime}(y-l), \psi_{0}\right\rangle+\cdots \\
& \equiv-l V^{\prime \prime \prime}\left(l^{\frac{4}{3}}\right) \psi_{0}(l)+l^{\frac{2}{3}} V^{\prime \prime}\left(l^{\frac{4}{3}}\right) \psi_{0}^{\prime}(l)+\cdots .
\end{aligned}
$$

Overall, using the asymptotics (5.13), (5.14) for the rescaled kernel and taking into account (6.15), we obtain from (6.19) the following typical approximate oscillatory behaviour:

$$
\lambda_{0}(l) \sim l^{\frac{2}{3}} \mathrm{e}^{-\hat{d}_{0} l^{4 / 3}} \cos \left(\hat{b}_{0} l^{\frac{4}{3}}\right) \quad \text { for } l \gg 1, \quad \hat{d}_{0} \sim d_{0}+b, \hat{b}_{0} \sim \frac{b_{0}+a}{2},
$$

where we omit constants. In fact, (6.20) shows how an $l$-branch of the first eigenvalue $\lambda_{0}(l)$ bifurcates at $l=+\infty$ from $\lambda_{0}(\infty)=0$ for the operator $\mathbf{B}^{*}$ with the spectrum (5.16), $m=2$. It is not that difficult to show that similar branching occurs from any eigenvalue

$$
\lambda_{k}=-\frac{k}{4} \quad \text { for } k=0,1,2, \ldots \Longrightarrow \exists \lambda_{k}(l) \quad \text { for } l \gg 1, \lambda_{k}(\infty)=-\frac{k}{4},
$$

where (6.20) is evidently the only eigenvalue that can change sign. The branching happens in the framework of classic perturbation theory for linear operators (see Kato [36]), so we do not treat this any further. The branching (6.21) or (6.10) is another reason for the non-self-adjoint operator (6.1) to admit infinitely many real eigenvalues $\left\{\lambda_{k}\right\}$.

\section{Bi-harmonic PDE for $m=2$ : blow-up asymptotic derivation of Petrovskii-type regularity criterion by eigenfunction expansion}

Regardless nonexistence of a definite answer concerning regularity of the vertex of the fundamental parabolae (4.13), nevertheless, we next show that there is a way to move towards a Petrovskii-like criterion for the case of more expanding lateral boundaries for functions (4.7). However, the results in Sect. 6 (treated according to the limit $l=l(\tau) \rightarrow+\infty)$ imply, in view of the oscillatory behaviour of the first eigenvalue in (6.20), that a direct regularity conclusion for a given $\varphi(\tau)$ is not possible in principle. To get the regular vertex $(0,0)$, a procedure of oscillatory cut off of the boundary must be performed. 
In Appendix, we will continue our study with a certain brief, rather questionable, and even controversial suggestion, which nevertheless is quite attractive and actually also goes along the lines of Petrovskii's barrier ideas. We fairly believe this is a right (and unique) place for such a discussion.

As a next step, we return to the spectral methods that cover both regular/ irregular issues for (4.1), which indeed are more complicated than for the heat equation (3.1).

\subsection{Two-region expansion}

Thus, we consider the rescaled problem (4.12) for $m=2$. As usual in any matching asymptotic analysis, this blow-up problem is solved by matching of expansions in two regions:

(i) Inner Region, which is situated around the origin $y=0$, and

(ii) Boundary Region close to the boundaries $y= \pm \varphi(\tau)$, where a boundary layer occurs.

Actually, such a two-region structure, with the asymptotics specified below, defines the class of generic solutions under consideration according to (4.3) (those in (4.4) are all different). We begin with the simpler analysis in the Boundary Region (ii).

\subsection{Boundary layer (BL) structure}

Sufficiently close to the lateral boundary of $Q_{0}$, it is natural to introduces the variables

$$
z=\frac{y}{\varphi(\tau)} \quad \text { and } \quad v(y, \tau)=w(z, \tau) \Longrightarrow w_{\tau}=-\frac{1}{\varphi^{4}} w_{z z z z}-\frac{1}{4} z w_{z}+\frac{\varphi^{\prime}}{\varphi} z w_{z} .
$$

We next introduce the BL-variables

$$
\xi=\varphi^{\frac{4}{3}}(\tau)(1-z), \quad \varphi^{\frac{4}{3}}(\tau) \mathrm{d} \tau=\mathrm{d} s, \quad \text { and } \quad w(z, \tau)=\rho(s) g(\xi, s),
$$

where $\rho(s)$ is an unknown slow decaying (in the same natural sense, associated with (4.8)) time-factor depending on the function $\varphi(\tau)$, e.g., $\sim \frac{1}{\varphi(\tau)}$ as a clue. On substitution into the PDE in (7.1), we obtain the following perturbed equation:

$$
\begin{aligned}
g_{s}= & \mathbf{A} g-\frac{1}{4} \varphi^{-\frac{4}{3}} \xi g_{\xi}-\frac{\varphi_{\tau}^{\prime}}{\varphi}\left(1-\xi \varphi^{-\frac{4}{3}}\right) g_{\xi} \\
& -\frac{4}{3} \varphi^{-\frac{1}{3}} \varphi_{\tau}^{\prime} \xi g_{\xi}-\frac{\rho_{s}^{\prime}}{\rho} g, \quad \text { where } \mathbf{A} g=-g^{(4)}+\frac{1}{4} g^{\prime} .
\end{aligned}
$$

As usual in boundary layer theory ${ }^{15}$ following (4.3), we are looking for a generic pattern of the behaviour described by (7.3) on compact subsets near the lateral boundary,

\footnotetext{
${ }^{15}$ It was always key for PDE theory. Shortly after the Blasius construction (1908) of the exact self-similar solution for the two-dimensional boundary layer equations proposed by Prandtl in 1904 (see references in [30, p. 48]), similarity solutions of linear and nonlinear boundary-value problems became more and more common in the literature.
} 


$$
|\xi|=o\left(\varphi^{-\frac{4}{3}}(\tau)\right) \Longrightarrow|z-1|=o\left(\varphi^{-\frac{8}{3}}(\tau)\right) \quad \text { as } \tau \rightarrow+\infty .
$$

On these space-time compact subsets, the second term on the right-hand side of (7.3) becomes asymptotically small, while all the others are much smaller in view of the slow growth/decay assumptions such as (4.8) for $\varphi(\tau)$ and $\rho(s)$.

Then posing the asymptotic behaviour at infinity:

$$
g(\xi, s) \rightarrow 1 \text { as } \xi \rightarrow+\infty \quad \text { (Hypothesis I for generic patterns), }
$$

where all the derivatives are assumed to vanish, we arrive at the problem of passing to the limit as $s \rightarrow+\infty$ in the problem (7.3), (7.5). Assuming that, by the definition in (7.2), the rescaled orbit $\{g(s), s>0\}$ is uniformly bounded, by classic parabolic theory [16], one can pass to the limit in (7.3) along a subsequence $\left\{s_{k}\right\} \rightarrow+\infty$. Namely, by the above, we have that, uniformly on compact subsets defined in (7.4), as $k \rightarrow \infty$,

$$
g\left(s_{k}+s\right) \rightarrow h(s), \quad \text { where } h_{s}=\mathbf{A} h, \quad h=h_{\xi}=0 \quad \text { at } \xi=0,\left.\quad h\right|_{\xi=+\infty}=1 .
$$

The limit (at $s=+\infty$ ) equation obtained from (7.3):

$$
h_{s}=\mathbf{A} h \equiv-h_{\xi \xi \xi \xi}+\frac{1}{4} h_{\xi}
$$

is a standard linear parabolic PDE in the unbounded domain $\mathbb{R}_{+}$, though it is governed by a non self-adjoint operator $\mathbf{A}$. We need to show that, in an appropriate weighted $L^{2}$-space if necessary and under the hypothesis (7.5), the stabilization holds, i.e., the $\omega$-limit set of $\{h(s)\}$ consists of equilibria: as $s \rightarrow+\infty$,

$$
\left\{\begin{array}{l}
h(\xi, s) \rightarrow g_{0}(\xi), \quad \text { where } \mathbf{A} g_{0}=0 \quad \text { for } \xi>0 \\
g_{0}=g_{0}^{\prime}=0 \text { for } \xi=0, \quad g_{0}(+\infty)=1 .
\end{array}\right.
$$

The characteristic equation for the linear operator $\mathbf{A}$ yields

$$
-\lambda^{4}+\frac{1}{4} \lambda=0 \Longrightarrow \lambda_{1}=0 \quad \text { and } \quad \lambda_{2,3}=\frac{1}{4^{1 / 3}}\left(-\frac{1}{2} \pm \mathrm{i} \frac{\sqrt{3}}{2}\right) \text {. }
$$

This gives the unique solution of (7.8), shown in Fig. 5,

$$
g_{0}(\xi)=1-\mathrm{e}^{-\frac{\xi}{2^{5 / 3}}}\left[\cos \left(\frac{\sqrt{3} \xi}{2^{5 / 3}}\right)+\frac{1}{\sqrt{3}} \sin \left(\frac{\sqrt{3} \xi}{2^{5 / 3}}\right)\right] .
$$

We do not concentrate on this stabilization problem (7.8), which reduces to a standard spectral study of $\mathbf{A}$ in a weighted space. Let us mention that, in the metric of $L^{2}\left(\mathbb{R}_{+}\right)$, the operator has a clear exponential stability feature. Consider the eigenvalue problem in an appropriate space of exponentially decaying functions,

$$
\mathbf{A} \psi \equiv-\psi^{(4)}+\frac{1}{4} \psi^{\prime}=\lambda \psi
$$




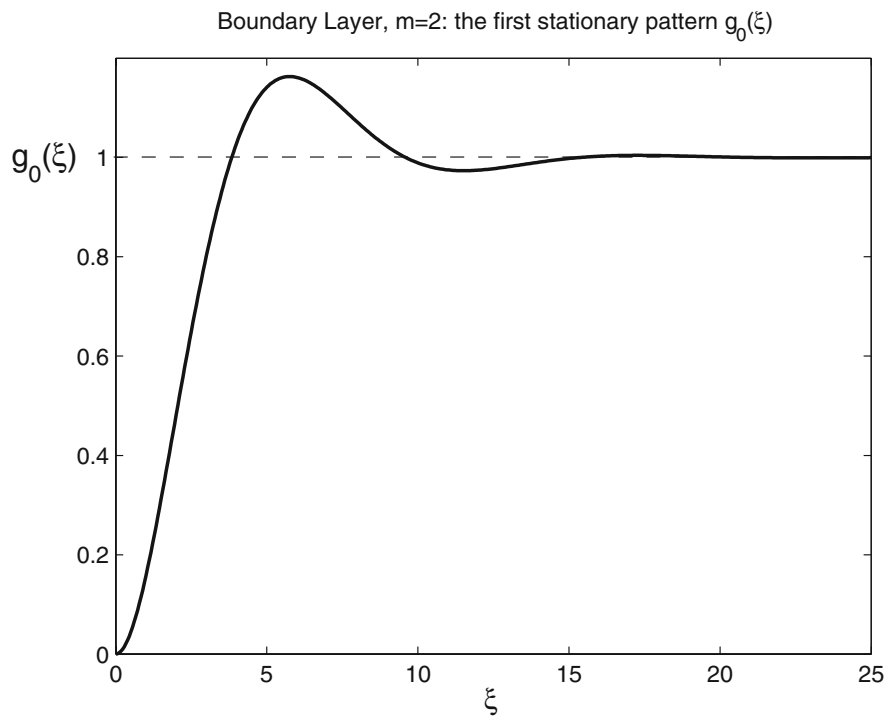

FiguRE 5. The unique stationary solution $g_{0}(\xi)$ of the problem (7.8)

As customary, multiplying this by $\bar{\psi}$, next taking the complex conjugate of the equation and multiplying by $\psi$ and summing up the resulting equalities yields

$$
(\lambda+\bar{\lambda}) \int|\psi|^{2}=-2 \int\left|\psi^{\prime \prime}\right|^{2}+\frac{1}{4} \int\left(\bar{\psi} \psi^{\prime}+\psi \bar{\psi}^{\prime}\right) \equiv-2 \int\left|\psi^{\prime \prime}\right|^{2}<0,
$$

i.e., $\operatorname{Re} \lambda<0$ for any $\lambda \in \sigma(\mathbf{A})$ (this does not take into account a continuous spectrum).

Actually, the convergence (7.6) and (7.8) for the perturbed dynamical system (7.3) is the main Hypothesis $(\mathrm{H})$, which characterizes the class of generic patterns under consideration, and then (7.5) is its partial consequence. Note that the uniform stability of the stationary point $g_{0}$ in the limit autonomous system (7.7) in a suitable metric will guarantee that the asymptotically small perturbations do not affect the omega-limit set; see [31, Chap. 1].

We stop further discussion concerning the passage to the limit in (7.3) and summarize the conclusions as follows:

Proposition 7.1. Under the given hypothesis and conditions, the problem (7.3) admits a family of solutions (called generic) that satisfy (7.8).

We must admit that such a definition of generic patterns looks rather non-constructive, which is unavoidable for higher-order parabolic PDEs without positivity issues. Note that, according to (3.14), a similar obscure issue appears even for the heat equation in (3.1). One can expect that (7.8) occurs for "almost all" solutions, excluding just those that have eventually the faster vanishing first Fourier coefficient. Then another BL is needed, but we do not intent to describe 
an invariant manifold structure of such a "thin" solution set (recall that (7.3) is a difficult non-autonomous PDE).

\subsection{Inner Region analysis: towards the regularity criterion}

In Inner Region, we deal with the original rescaled problem (4.12). Without loss of generality, for simplicity of key calculations, we consider symmetric solutions defined for $y>0$ by assuming the symmetry conditions:

$$
v_{y}=v_{y y y}=0 \quad \text { at } \quad y=0 .
$$

In order to apply the standard eigenfunction expansion techniques by using the orthonormal set of polynomial eigenfunctions of $\mathbf{B}^{*}$ given in $(5.20)$, as customary in classic PDE and potential theory, we extend $v(y, \tau)$ by 0 for $y>\varphi(\tau)$ :

$$
\hat{v}(y, \tau)=v(y, \tau) H(\varphi(\tau)-y)= \begin{cases}v(y, \tau) & \text { for } 0 \leq y<\varphi(\tau) \\ 0 & \text { for } y \geq \varphi(\tau)\end{cases}
$$

where $H$ is the Heaviside function. Since $v=v_{y}=0$ on the lateral boundary $\{y=\varphi(\tau)\}$, one can check that, in the sense of distributions,

$$
\begin{gathered}
\hat{v}_{\tau}=v_{\tau} H, \quad \hat{v}_{y}=v_{y} H, \quad \hat{v}_{y y}=v_{y y} H, \\
\hat{v}_{y y y}=v_{y y y} H-\left.v_{y y}\right|_{y=\varphi} \delta(y-\varphi), \\
\hat{v}_{y y y y}=v_{y y y y} H-\left.v_{y y y}\right|_{y=\varphi} \delta(y-\varphi)-\left.v_{y y}\right|_{y=\varphi} \delta^{\prime}(y-\varphi) .
\end{gathered}
$$

Therefore, $\hat{v}$ satisfies the following equation:

$$
\hat{v}_{\tau}=\mathbf{B}^{*} \hat{v}-\left.v_{y y y}\right|_{y=\varphi} \delta(y-\varphi)-\left.v_{y y}\right|_{y=\varphi} \delta^{\prime}(y-\varphi) \quad \text { in } \mathbb{R}_{+} \times \mathbb{R}_{+} .
$$

Since, obviously, the extended solution orbit (7.14) is uniformly bounded in $L_{\rho^{*}}^{2}(\mathbb{R})$, we can use the converging in the mean (and uniformly on compact subsets in $y$ ) the eigenfunction expansion via the generalized Hermite polynomials (5.20):

$$
\hat{v}(y, \tau)=\sum_{(k \geq 0)} a_{k}(\tau) \psi_{k}^{*}(y) .
$$

Substituting (7.17) into (7.16) and using the orthonormality property (5.21) yields the following dynamical system for the expansion coefficients: for all $k=0,1,2, \ldots$,

$$
a_{k}^{\prime}=\lambda_{k} a_{k}-\left.v_{y y y}\right|_{y=\varphi(\tau)}\left\langle\delta(y-\varphi(\tau)), \psi_{k}\right\rangle-\left.v_{y y}\right|_{y=\varphi(\tau)}\left\langle\delta^{\prime}(y-\varphi), \psi_{k}\right\rangle,
$$

where $\lambda_{k}=-\frac{k}{4}$ are real eigenvalues (5.16). Recall that $\lambda_{k}<0$ for all $k \geq 1$. More importantly, the corresponding eigenfunctions $\psi_{k}(y)$ are unbounded and not monotone for $k \geq 1$ according to (5.22). Therefore, regardless proper asymptotics given by (7.18), these inner patterns cannot be matched with the BL-behaviour such as (7.5), and demand other matching theory. Since these are not generic, the latter is not developed.

Thus, bearing again in mind (4.3), one needs to concentrate on the "maximal" first Fourier generic pattern associated with

$$
k=0: \quad \lambda_{0}=0 \quad \text { and } \quad \psi_{0}^{*}(y) \equiv 1 \quad\left(\psi_{0}(y)=F(y)\right) .
$$


Actually, this corresponds to a naturally understood "centre subspace behaviour" for the Eq. (7.18):

$$
\hat{v}(y, \tau)=a_{0}(\tau) \cdot 1+w^{\perp}(y, \tau), \quad \text { where } w^{\perp} \in \operatorname{Span}\left\{\psi_{k}^{*}, k \geq 1\right\},
$$

and $w^{\perp}(y, \tau)$ is then negligible relative to $a_{0}(\tau)$. This is another characterization of our class of generic patterns, Hypothesis II. The equation for $a_{0}(\tau)$ then takes the form:

$$
a_{0}^{\prime}=-\left.v_{y y y}\right|_{y=\varphi(\tau)} \psi_{0}(\varphi(\tau))+\left.v_{y y}\right|_{y=\varphi(\tau)} \psi_{0}^{\prime}(\varphi(\tau)) .
$$

We now return to BL theory established the boundary behaviour (7.2) for $\tau \gg 1$, which for convenience we state again: in the rescaled sense, on the given compact subsets,

$$
v(y, \tau)=\rho(s) g_{0}\left(\varphi^{\frac{4}{3}}(\tau)\left(1-\frac{y}{\varphi(\tau)}\right)\right)+\cdots .
$$

By the matching of both Regions, one concludes that, for such generic patterns,

$$
\frac{a_{0}(\tau)}{\rho(s)} \rightarrow 1 \quad \text { as } \tau \rightarrow \infty \Longrightarrow \rho(s)=a_{0}(\tau)(1+o(1)) .
$$

Then the convergence (7.22), which by a standard parabolic regularity is also true for the spatial derivatives, yields, in the natural rescaled sense,

$$
\begin{gathered}
\left.v_{y y}\right|_{y=\varphi(\tau)} \rightarrow \rho(s) \varphi^{\frac{2}{3}}(\tau) \gamma_{1} \rightarrow a_{0}(\tau) \varphi^{\frac{2}{3}}(\tau) \gamma_{1}, \quad \gamma_{1}=g_{0}^{\prime \prime}(0)>0, \\
\left.v_{y y y}\right|_{y=\varphi(\tau)} \rightarrow-\rho(s) \varphi(\tau) \gamma_{2} \rightarrow-a_{0}(\tau) \varphi(\tau) \gamma_{2}, \quad \gamma_{2}=g_{0}^{\prime \prime \prime}(0) .
\end{gathered}
$$

Eventually, this leads to the following asymptotic ODE for the first expansion coefficient for generic patterns: ${ }^{16}$

$$
\frac{a_{0}^{\prime}}{a_{0}}=G_{2}(\varphi(\tau)) \equiv \gamma_{2} \varphi(\tau) \psi_{0}(\varphi(\tau))+\gamma_{1} \varphi^{\frac{2}{3}}(\tau) \psi_{0}^{\prime}(\varphi(\tau))+\cdots \quad \text { for } \tau \gg 1 .
$$

For instance, this gives a first easy condition of regularity of $(0,0)$ : for the generic patterns, this is the "negative" divergence of the following integral:

$$
\int^{\infty} G_{2}(\varphi(\tau)) \mathrm{d} \tau \text { diverges to }-\infty \Longrightarrow a_{0}(\tau) \rightarrow 0 \quad \text { as } \tau \rightarrow+\infty .
$$

In order to obtain more practical and sufficiently sharp conditions of regularity, we will use the expansion (5.13) of the first eigenfunction $\psi_{0}(y)=F(y)$, which on substitution into the right-hand side in (7.25), where both terms are equivalent, yields

$$
\frac{a_{0}^{\prime}}{a_{0}}=\hat{\gamma} \varphi^{\frac{2}{3}}(\tau) C_{3} \cos \left(b_{0} \varphi^{\frac{4}{3}}(\tau)+C_{4}\right) \mathrm{e}^{-d_{0} \varphi^{4 / 3}(\tau)}+\cdots \quad \text { for } \tau \gg 1,
$$

\footnotetext{
${ }^{16}$ This result can be stated as a theorem: For the prescribed above class of generic solutions, the first Fourier coefficient satisfies...; as we have mentioned, we avoid such non-constructive, but rigorous, ones.
} 
with some $\hat{\gamma} \neq 0$ and constants $C_{3,4}$ depending in an obvious way on $C_{1,2}$ in (5.13) and other parameters from (5.14). Integrating implies that

$$
\ln \left|a_{0}(\tau)\right|=\hat{\gamma} \int^{\tau} \varphi^{\frac{2}{3}}(s) C_{1} \cos \left(b_{0} \varphi^{\frac{4}{3}}(s)+C_{2}\right) \mathrm{e}^{-d_{0} \varphi^{4 / 3}(s)} \mathrm{d} s+\cdots \quad \text { for } \tau \gg 1 .
$$

The regularity condition (7.26) then to be re-formulated according to (7.28).

Irregularity condition. This is straightforward: (7.28) implies that the limit of $a_{0}(\tau)$ as $\tau \rightarrow+\infty$ can be arbitrary (i.e., not necessarily zero), if the integral converges. Therefore, up to the achieved accuracy of the expansions and matching, we state the following condition of the irregularity of $(0,0)$, where all the irrelevant constants are omitted:

$(0,0)$ is irregular if $\int^{\infty} \varphi^{\frac{2}{3}}(s) \cos \left(b_{0} \varphi^{\frac{4}{3}}(s)\right) \mathrm{e}^{-d_{0} \varphi^{4 / 3}(s)} \mathrm{d} s$ converges.

Then, obviously, $u\left(0,0^{-}\right) \neq 0$ (a natural non-orthogonality condition required) is finite, i.e., the vertex $(0,0)$ is irregular, but non-singular. This still looks pretty similar to Petrovskii's criterion in (3.11).

Regularity condition: oscillatory cut-off of $\varphi(\tau)$. Thus, assume that $\varphi(\tau)$ is close to the desired (and still unknown) critical irregularity/regularity situation, and that we follow the first Fourier coefficient equation (7.27) or (7.28), within the given accuracy. Then, for using (7.28) for the purpose of the regularity analysis, we observe that the presence in the integral the oscillatory factor $\cos (\cdot)$ can always violate the (uniform) divergence to $-\infty$ hypothesis in (7.26). Indeed, then $\ln \left|a_{0}(\tau)\right| \rightarrow \pm \infty$ along different subsequences $\left\{\tau=\tau_{k}^{ \pm}\right\} \rightarrow+\infty$, and the limit to $+\infty$ does not guarantee (7.26). In its turn, of course, for such limits, our matching with the BL can be violated as well, but we do not discuss this issue here. In other words, in the case of oscillatory kernels,

a pure divergence of the integral in (7.28) does not guarantee the point regularity.

This is the principal difference with the positive kernel case. Cf. (7.36) for $m=1$ below with no oscillatory component in the integral. Let us note that, in general, the standard divergence of (7.28) will ensure that the vertex $\left(0,0^{-}\right)$is irregular and is also singular in the sense of (4.15). Moreover, $u(x, t)$ has infinite oscillatory behaviour as $(x, t) \rightarrow\left(0,0^{-}\right)$, i.e., eventually oscillations get both $\pm \infty$.

Thus, under the accepted hypothesis, the oscillatory part of the rescaled fundamental kernel (5.13) of the operator under consideration can violate the regularity of boundary point for any slow growing factor $\varphi(\tau)$ satisfying (4.7)-(4.9), even if the integral diverges. As we have seen in Sect. 6, for higher-order operators, the regularity condition "above" the fundamental parabolae (4.13) becomes rather subtle and sensitive. Indeed, according to the integral in (7.28), arbitrarily 
small perturbations of $\varphi(\tau)$ can "switch over" regularity to the irregularity and vice versa.

To guarantee regularity, an extra "oscillatory cut-off" of $\varphi(\tau)$ is then necessary to be introduced after a necessary remark.

\subsection{Remark 1: an analogy with elliptic theory}

This principal difficulty concerning the kernels of changing sign has a known counterpart in regularity elliptic theory for (2.1). Namely, it was first shown in [64] (1986) for $m=2, N \geq 8$ that the vertex of a cone can be irregular (singular) if the fundamental solution of $L(\partial)$ for $N>2 m^{17}$

$$
F(x)=\Psi\left(\frac{x}{|x|}\right)|x|^{2 m-N}, \quad x \in \mathbb{R}^{N} \backslash\{0\} \quad(N>2 m)
$$

changes sign; see further comments and references in [63, Sect. 1]. Then in elliptic theory, the regularity analysis is performed for a restricted subclass of operators $L(\partial)$ called positive with weight $F$. This actually means that $F>0[63$, Sect. 3] (cf. a positivity-like condition in [24] for $m=2$ and [18]), so, until now, refined elliptic regularity results for kernels of essentially (e.g., infinitely many) changing sign seem to be unavailable. As in classic theory [44,65], this analysis demands constructing corresponding generalized Hermite polynomials as eigenfunctions of an adjoint pencil of linear operators along the lines of those obtained for hyperbolic equations; see [30, p. 254] and [26] (for $u_{t t}=-u_{x x x x}$; see the end of Sect. 8 below). The oscillatory cut-off of the boundary will then be essential. An example of such an elliptic evolution approach for studying singularities of $(2.2)$ in $\mathbb{R}^{2}$, $q \in(0,1)$ (with many additional references presented) is given in [9].

Of course, it is well-known and this is a classic matter, that general theory of operator pencils associated with corner singularities has been well-developed for a number of elliptic equations; see $[47,48]$ and [24] for references to other papers and related monographs and as a source of further extensions to be traced out further by the MathSciNet. For other types of parabolic or elliptic problems mentioned above, new operator pencils appear. In the parabolic case, we do not intend to change the operator $-D_{x}^{4}$ in related lines, ${ }^{18}$ but then need instead to perform a refined oscillatory cut-off of the lateral boundary.

${ }^{17}$ For $N=2 m$, the structure of the fundamental solution is different and more signed-determined:

$$
F(x)=\kappa \ln \frac{1}{|x|}+\Psi\left(\frac{x}{|x|}\right) \quad(\kappa(N)=\text { const. }),
$$

that makes the case special admitting arbitrary operators $L(\partial)$ [63, Sect. 8]; Wiener's $m=1$, $N=2$ included.

${ }^{18}$ Obviously, unlike elliptic theory, this is not possible: no higher-order parabolic equations with smooth coefficients can have positive kernels (otherwise, comparison and the MP would be inherited). 


\subsection{Optimal regularity conditions for generic patterns}

Thus, to guarantee the regularity, we perform the procedure of oscillatory cut-off of the given boundary function $\varphi(\tau)$. Namely, this means that a smooth $\tilde{\varphi}(\tau)$ replaces $\varphi(\tau)$, for which

$$
\hat{\gamma} C_{1} \cos \left(b_{0} \widetilde{\varphi}^{\frac{4}{3}}(\tau)+C_{2}\right) \approx\left[\hat{\gamma} C_{1} \cos \left(b_{0} \varphi^{\frac{4}{3}}(\tau)+C_{2}\right)\right]_{-}
$$

asymptotically sharp up to absolutely convergent perturbations in the integral (7.28), where $[\cdot]_{-}$denotes the negative part. This is necessary to cut-off the positive part of the diverging integral. Figure 6 schematically explains how (7.31) works (for $\hat{\gamma} C_{1}>0$ ) by cutting off all positive waves of the $\cos$-function for $\tau \gg 1$ and creating "almost discontinuous" (jumping-like) $\widetilde{\varphi}(\tau)$, which in the figure is denoted by $\varphi_{\text {cut }}(\tau)$. Using necessary smoothing at the points, where $\cos (\cdot)$ changes sign, for any such monotone increasing $\varphi(\tau)$, the corresponding $\widetilde{\varphi}(\tau)$ can be also chosen increasing. In other words, the resulting $\widetilde{\varphi}(\tau)$ sufficiently fast jumps over those intervals of the length $l$, which in Sect. 6 were determined as leading to the irregular vertex. We do not pay special attention to the question on how such smoothly jumping boundary can affect the boundary layer behaviour described by the Eq. (7.3), where the term $\varphi^{\prime} / \varphi$ may then play a role (fortunately, not dominant). However, it is clear that, since the correct intervals of the behaviour of $\widetilde{\varphi}(\tau)$ get arbitrarily long as $\tau \rightarrow \infty$, we have enough time for establishing the BL-structure, so that formulae (8.5) remain true closer to the end of each of them, and further analysis applies. In addition, it is also clear that, once the solution gets very small by (7.25) at the end of a fixed such interval in $\tau$, a smooth monotone increasing jump of $\widetilde{\varphi}(\tau)$ at the end point cannot essentially affect the value of the solution during such a short interval of time. This means that, on the next good interval, the solution remains small and continues to be governed by the eigenspace Span $\left\{\psi_{0}^{*}\right\}$.

Thus, according to the oscillatory cut-off (7.31), the condition of regularity is as follows:

$$
(0,0) \text { is regular if } \int^{\infty} \widetilde{\varphi}^{\frac{2}{3}}(s) \cos \left(b_{0} \widetilde{\varphi}^{\frac{4}{3}}(s)+C_{2}\right) \mathrm{e}^{-d_{0} \widetilde{\varphi}^{4 / 3}(s)} \mathrm{d} s \text { diverges. }
$$

There is a clear gap between the irregularity (7.29) and the regularity (7.32) conditions, so that a pure Osgood-Dini-like criterion cannot be available in principle.

With such a procedure, similar to Petrovskii's presentation (3.9) of the boundary regularity results, we deduce that:

(i) $\widetilde{R}(t)=3^{-\frac{3}{4}} 2^{\frac{11}{4}}(-t)^{\frac{1}{4}}[\ln |\ln (-t)|]^{\frac{3}{4}} \Longrightarrow(0,0)$ is regular, and

(ii) $R(t)=\left(3^{-\frac{3}{4}} 2^{\frac{11}{4}}+\varepsilon\right)(-t)^{\frac{1}{4}}[\ln |\ln (-t)|]^{\frac{3}{4}}, \varepsilon>0 \Longrightarrow(0,0)$

is irregular,

where, in the regular $\widetilde{R}(t)$, the oscillatory cut-off meaning (7.31) must be assumed. 


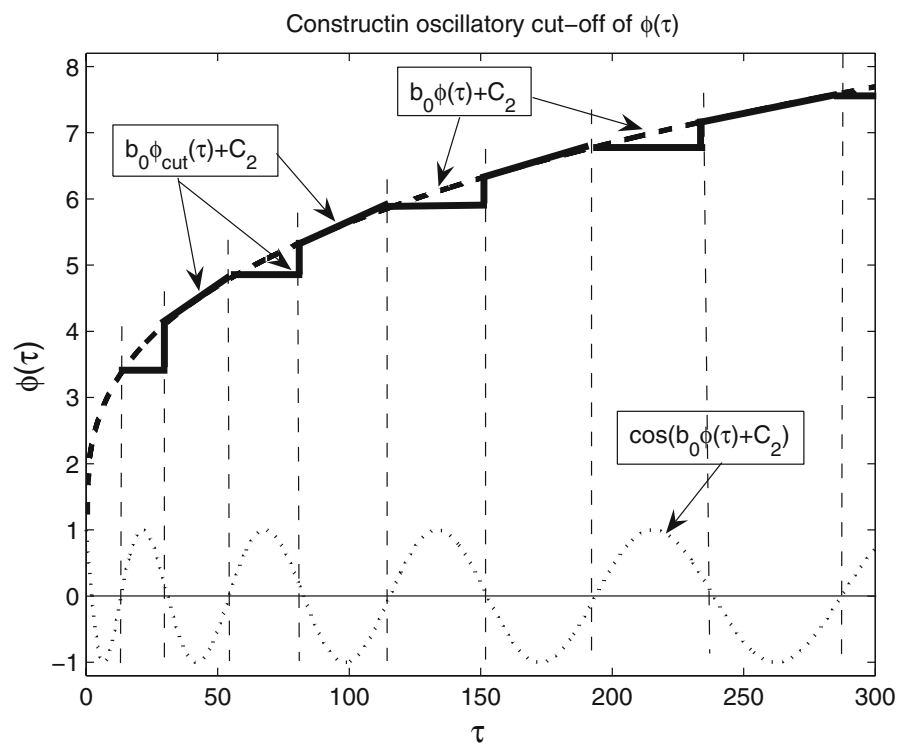

FiguRE 6. A schematic view of a proper oscillatory cut-off of $\varphi(\tau)$

\subsection{Remark 2: on other asymptotic patterns and related regularity}

It follows from the dynamical system (7.18), that, in general, there exist other (non-generic) asymptotic patterns corresponding to the behaviour on each of a 1D stable eigenspace of $\mathbf{B}^{*}$, which hence are not governed by the expansion (7.20). Such a behaviour then will generate its own regularity/irregularity conditions with or without cut-offs of the corresponding similar integrals. Indeed, those asymptotic patterns will demand a different "BL-like" theory, for which (7.5) is not true. Since these are not generic and belong to the subspace of co-dimension one, a correct posing of the IBVP in $Q_{0}$ for such regular solutions is quite tricky and demands a priori unknown and non-constructive conditions on initial data $u_{0}$, so we have no reasons to take those into account. On the other hand, describing countable sets of various blow-up singularities is a serious problem of modern PDE theory, but currently this has a little to do with the boundary regularity analysis.

\subsection{Remark 3: for the heat equation, the spectral-BL blow-up approach is sharp}

Let us very briefly repeat and list the main steps of the above blow-up analysis for the classic problem (3.1) to prove (4.5). Thus, (4.6) reads

$$
R(t)=(-t)^{\frac{1}{2}} \varphi(\tau),
$$


where, in (4.12), we get the classic Hermite operator [10, p. 48]

$$
\begin{aligned}
& \mathbf{B}^{*}=D_{y}^{2}-\frac{1}{2} y D_{y} \quad \text { in } L_{\rho^{*}}^{2}(\mathbb{R}), \quad \rho^{*}(y)=\mathrm{e}^{-\frac{y^{2}}{4}}, \\
& \sigma\left(\mathbf{B}^{*}\right)=\left\{\lambda_{k}=-\frac{k}{2}, k=0,1,2, \ldots\right\},
\end{aligned}
$$

with $\mathcal{D}\left(\mathbf{B}^{*}\right)=H_{\rho^{*}}^{2}(\mathbb{R})$, etc. Similar to $(7.2)$, the BL variables and asymptotics are now

$$
\begin{aligned}
& \xi=\varphi^{2}(1-z) \equiv \varphi(\varphi-y) \quad \text { and } \quad w(z, \tau)=a_{0}(\tau) g(\xi, \tau)+\cdots \\
& \text { where } g(\xi, \tau) \rightarrow g_{0}(\xi), \tau \rightarrow \infty \text { and } g_{0}(\xi)=1-\mathrm{e}^{-\xi / 2} \quad\left(g_{0}^{\prime \prime}+\frac{1}{2} g_{0}^{\prime}=0\right) .
\end{aligned}
$$

Using two formulae in the first line in (7.15) with

$$
\hat{v}_{y y}=v_{y y} H-\left.v_{y}\right|_{y=\varphi} \delta(y-\varphi),
$$

we arrive at the equation (the analogy of (7.16))

$$
\hat{v}_{\tau}=\mathbf{B}^{*} \hat{v}+\left.v_{y}\right|_{y=\varphi} \delta(y-\varphi) .
$$

Hence, asymptotically via the BL structure (7.34), for the generic patterns as usual, the analogy of (8.6) takes much simpler form:

$$
\frac{a_{0}^{\prime}}{a_{0}}=-\gamma_{1} \varphi \psi_{0}(\varphi)+\cdots \Longrightarrow \frac{a_{0}^{\prime}}{a_{0}}=-\frac{\gamma_{1}}{2 \sqrt{\pi}} \varphi \mathrm{e}^{-\frac{\varphi^{2}}{4}}+\cdots,
$$

where $\gamma_{1}=g_{0}^{\prime}(0)=\frac{1}{2}$ and $\psi_{0}(y)=F(y)=\frac{1}{2 \sqrt{\pi}} \mathrm{e}^{-y^{2} / 4}$ is the positive rescaled Gaussian kernel (5.11) of the heat operator. Thus, (7.35) justifies that, for the generic patterns, the regularity criterion reads

$$
(0,0) \text { is regular iff } \int^{\infty} \varphi(\tau) \mathrm{e}^{-\frac{\varphi^{2}(\tau)}{4}} \mathrm{~d} \tau=+\infty,
$$

with no cut-off in this non-oscillatory case. One can see that (7.36) is equivalent to that in (3.11) or (3.12). Recall that there exist other asymptotic patterns related to the stable subspace of $\mathbf{B}^{*}$ of co-dimension 1 , which, as usual, is not taken into account.

\section{On extensions to $2 m$ th-order poly-harmonic and other operators}

We next discuss the extensions of the asymptotic method to other PDEs.

\subsection{Poly-harmonic equation}

For (1.4), the spectral properties of the operator

$$
\mathbf{B}^{*}=-(-\Delta)^{m}-\frac{1}{2 m} y \cdot \nabla
$$


are given in [15]. A sharp asymptotic expansion (similar to (5.13)) of the radially symmetric rescaled kernel $F(|y|)$ satisfying

$$
\mathbf{B} F=0 \quad \text { in } \mathbb{R}^{N}, \quad \int F=1 \Longrightarrow \psi_{\beta}(y)=\frac{(-1)^{|\beta|}}{\sqrt{\beta !}} D^{\beta} F(y),|\beta|=0,1,2, \ldots,
$$

where $\beta$ is a multiindex in $\mathbb{R}^{N}$, is also not that difficult to get. Then (8.2) implies that the adjoint basis $\left\{\psi_{\beta}^{*}\right\}$ consists of generalized Hermite polynomials.

In the radial case, the critical $R(t)$ for boundary regularity is about

$$
R(t)=C_{*}(-t)^{\frac{1}{2 m}}[\ln |\ln (-t)|]^{\frac{2 m-1}{2 m}}, \quad \text { where } C_{*}=d_{0}^{-\frac{1}{\alpha}}>0 \text { is a constant, }
$$

and the oscillatory cut-off is assumed for the regularity. As usual, replacing $C_{*}$ by $C_{*}+\varepsilon$ for any $\varepsilon>0$ makes $(0,0)$ irregular.

Some calculations become more involved, especially in the case of non-radial $Q_{0}$, where the spatial shape of the shrinking cusp will affect the right-hand side of (7.27). Hence, the BL structure, though having similar variables such as (7.2), is now governed by complicated elliptic problems instead of the ODE (7.11). For instance, let the boundary of $Q_{0}$ be given by the characteristic fundamental paraboloid in the $x$ and $y$ variables, respectively (i.e., $\varphi(\tau) \equiv 1$ ):

$$
\sum_{(|\alpha|=2 m)} a_{\alpha} x^{\alpha}=(-t) \Longrightarrow \sum_{(|\alpha|=2 m)} a_{\alpha} y^{\alpha}=1
$$

The case $a_{\alpha}>0$ for any $\alpha$ corresponds to Mihailov's [66-68] and Kondrat'ev's [43] cases. Hence, the regularity in the strong sense of the vertex $(0,0)$ depends on the spectrum of the operator (8.1) in the domain $I_{a}$ with the boundary given by (8.4). Similar to the analysis in Sect. 6 , we have that, by Poincaré's inequality, $(0,0)$ is regular if the diameter of $I_{a}$ is not that large (since the eigenvalues of $\mathbf{B}^{*}$ are close to those for the self-adjoint $\left.-(-\Delta)^{m}<0\right)$. On the contrary, since $\mathbf{B}^{*}$ is not self-adjoint and/or sign-definite, there are coefficients $\left\{a_{\alpha}>0\right\}$, for which the vertex $(0,0)$ is not regular in the classic sense.

If some of the coefficients $\left\{a_{\alpha}\right\}$ are negative (this is Feigin's case [22]), $\mathbf{B}^{*}$ is then posed in an unbounded domain with a non-compact boundary, and its spectral theory becomes more involved. Nevertheless, for sufficiently "thin" domains, we may expect the vertex be regular, and sometimes irregular otherwise.

\subsection{Linear dispersion equations}

There is no much difference in studying the boundary regularity for odd-order linear PDEs. For instance, as a simple example, consider the third-order linear dispersion equation in a similar setting:

$$
u_{t}=u_{x x x} \quad \text { in } Q_{0}=\left\{-R_{-}(t)<x<R_{+}(t), \quad-1<t<0\right\},
$$


where $R_{ \pm}(t)$ are positive smooth functions on $[-1,0)$, continuous on $[-1,0]$, and $R_{ \pm}(0)=0$. Since the operator $D_{x}^{3}$ is anisotropic, the functions $R_{ \pm}(t)$ are essentially different. According to odd-order PDE theory (see e.g., [21]), for (8.5), it is allowed to put the following Dirichlet boundary conditions:

$$
u=u_{x}=0 \quad \text { at } x=R_{-}(t) \quad \text { and } \quad u=0 \quad \text { at } x=R_{+}(t), \quad t \in[-1,0) .
$$

Since, as we will show, the main singular phenomena occur at the right-hand "oscillatory" lateral boundary $\left\{x=R_{+}(t)\right\}$, we concentrate on this analysis, and, in general, can put

$$
R_{-}(t) \equiv-\infty,
$$

so excluding this part of the boundary from consideration. Then, the regularity criterion of $(0,0)$ will solely depend on the behaviour of $R_{+}(t)$ as $t \rightarrow 0^{-}$.

Thus, taking into account the right-hand lateral boundary, as in (4.6), we perform the first rescaling:

$$
\begin{aligned}
& R_{+}(t)=(-t)^{\frac{1}{3}} \varphi_{+}(\tau), \quad y=\frac{x}{(-t)^{1 / 3}}, \quad \tau=-\ln (-t), \quad u(x, t)=v(y, \tau), \\
& \text { where } v_{\tau}=\mathbf{B}^{*} v \equiv v_{y y y}-\frac{1}{3} y v_{y}, \quad v=0 \quad \text { at } y=\varphi_{+}(\tau), \tau \geq 0 .
\end{aligned}
$$

Hence, unlike parabolic equations, for (8.5), another (but indeed similar in many places) version of Hermitian spectral theory for $\mathbf{B}^{*}$ is necessary to get optimal behaviour of $R_{+}(t)$ as $t \rightarrow 0^{-}$for the regularity/irregularity of $(0,0)$.

First, as above, the regularity criterion is associated with the structure of the fundamental solution of (8.5) that has the similarity form

$$
b(x, t)=t^{-\frac{1}{3}} F(y), \quad y=\frac{x}{t^{1 / 3}},
$$

where $F=\operatorname{Ai}(y)$ is the Airy function satisfying

$$
\mathbf{B} F \equiv F^{\prime \prime \prime}+\frac{1}{3}(y F)^{\prime}=0 \quad \text { in } \mathbb{R}, \quad \int F=1 \Longrightarrow F^{\prime \prime}+\frac{1}{3} y F=0 .
$$

Hence, the rescaled kernel $F(y)$ has an exponential decay as $y \rightarrow-\infty$ only, and as $y \rightarrow+\infty$ is oscillatory according to the two-scale WKBJ-type asymptotics

$$
F(y)=\left\{\begin{array}{l}
C_{1}|y|^{-\frac{1}{4}} \mathrm{e}^{-d_{0}|y|^{3 / 2}}+\cdots \quad \text { as } y \rightarrow-\infty \\
y^{-\frac{1}{4}}\left[C_{2} \cos \left(d_{0} y^{\frac{3}{2}}\right)+C_{3} \sin \left(d_{0} y^{\frac{3}{2}}\right)\right]+\cdots \quad \text { as } y \rightarrow+\infty
\end{array}\right.
$$

where $d_{0}=\frac{2 \sqrt{3}}{9}$ and $C_{1,2,3}$ are some constants of non-essential values.

Secondly, this (see extra details in [25, Sect. 9.2] and [23]) generates very oscillatory as $y \rightarrow+\infty$ and unbounded (for any $k \geq 1$ ) eigenfunctions of the linear operator $\mathbf{B}$ given in (8.10) (cf. (5.17))

$$
\psi_{k}(y)=\frac{(-1)^{k}}{\sqrt{k !}} D_{y}^{k} F(y) \quad(F=\operatorname{Ai}(y)), \quad \text { with } \sigma(\mathbf{B})=\left\{-\frac{k}{3}, k=0,1,2, \ldots\right\} .
$$


For existence of such a discrete spectrum, a proper "radiation" condition at $y=$ $+\infty$ is posed for functions from the domain of $\mathbf{B}$, which excludes "non-oscillatory" (polynomial-rational) bundles that are different from those in (8.11). The "adjoint" linear operator

$$
\mathbf{B}^{*}=D_{y}^{3}-\frac{1}{3} y D_{y}, \quad \text { with } \quad \sigma\left(\mathbf{B}^{*}\right)=\sigma(\mathbf{B})=\left\{-\frac{k}{3}, k=0,1,2, \ldots\right\},
$$

has a complete set of eigenfunctions $\left\{\psi_{k}^{*}\right\}$ that are generalized Hermite polynomials. In general, then the bi-orthonormality property (5.21) demands using Hahn-Banach Theorem on extensions of continuous linear functionals (or similar techniques of regularization of oscillatory integrals in a v.p. or c.r. sense) and other constructions. This is not that crucial at the moment for our particular Fourier-type analysis that involves the first mode only. Both operators are defined in special weighted $L^{2}$ spaces. One can see that $\mathbf{B}^{*}$ is not adjoint to $\mathbf{B}$ in the standard metric of $L^{2}$, since, obviously, then $\tilde{\mathbf{B}}^{*}=-\mathbf{B}+\frac{1}{3} I$, so $\mathbf{B}-\frac{1}{6} I$ is skew-symmetric. In fact, $\mathbf{B}^{*}$ is adjoint to $\mathbf{B}$ in a space with the indefinite metric given by

$$
\langle v, w\rangle_{*}=\int v(y) \overline{w(-y)} \mathrm{d} y .
$$

This case corresponds to the decomposable space with indefinite metric with straightforward majorizing one and is treated as rather trivial; see AzizovIokhvidov [6] for linear operators theory in spaces with indefinite metric (the theory was initiated in the 1940 s and 50 s by Pontryagin and Krein). Then the domain of $\mathbf{B}^{*}$ is defined as $H_{\rho^{*}}^{3}$, etc.

Thus, it is key that the bounded operator $\mathbf{B}^{*}: H_{\rho^{*}}^{3} \rightarrow L_{\rho^{*}}^{2}$ admits a complete set of polynomial eigenfunctions $\Phi^{*}=\left\{\psi_{k}^{*}(y)\right\}$, which are constructed similarly to those obtained in (5.20). Quite analogously, these polynomials are used in our regularity analysis.

Boundary Layer at $y=R_{+}(t)$. Similar to (7.1), (7.2), the BL-variables are

$$
z=\frac{y}{\varphi_{+}(\tau)}, \quad \xi=\varphi_{+}^{\frac{3}{2}}(\tau)(1-z), \quad w(z, \tau)=\rho(\tau) g(\xi, \tau)
$$

This yields the asymptotic problem of stabilization to a unique profile $g_{0}(\xi)$ (as usual, we omit this not that easy analysis), where, as seen from (8.8),

$$
g_{0}^{\prime \prime \prime}-\frac{1}{3} g_{0}^{\prime}=0, g_{0}(0)=1, g_{0}(+\infty)=1 \Longrightarrow g_{0}(\xi)=1-\mathrm{e}^{-\xi / \sqrt{3}} .
$$

Inner Region: the regularity criterion for $R_{+}(t)$. Using the formulae as in (7.15), we obtain here the equation

$$
\hat{v}_{\tau}=\mathbf{B}^{*} \hat{v}+\left.v_{y y}\right|_{y=\varphi_{+}} \delta\left(y-\varphi_{+}\right)+\left.v_{y}\right|_{y=\varphi_{+}} \delta^{\prime}\left(y-\varphi_{+}\right) \quad \text { in } \mathbb{R} \times \mathbb{R}_{+},
$$

and use the eigenfunction expansion (7.17) via the corresponding generalized Hermite polynomials that leads to the dynamical system such as (7.18). Looking for the generic patterns with the representation (7.20), (8.17) yields 


$$
a_{0}^{\prime}=\left.v_{y y}\right|_{y=\varphi_{+}(\tau)} \psi_{0}\left(\varphi_{+}(\tau)\right)-\left.v_{y}\right|_{y=\varphi_{+}(\tau)} \psi_{0}^{\prime}\left(\varphi_{+}(\tau)\right) \quad\left(\psi_{0}(y) \equiv F(y)\right) .
$$

By BL theory and matching, we can use the fact that, in the rescaled sense, on the given compact subsets, there is the convergence, with first derivatives:

$$
v(y, \tau)=a_{0}(\tau) g_{0}\left(\varphi_{+}^{\frac{3}{2}}(\tau)\left(1-\frac{y}{\varphi_{+}(\tau)}\right)\right)+\cdots .
$$

Eventually, this leads to the following asymptotic ODE for the first expansion coefficient for generic patterns:

$$
\frac{a_{0}^{\prime}}{a_{0}}=G_{3}\left(\varphi_{+}(\tau)\right) \equiv \gamma_{2} \varphi_{+}(\tau) \psi_{0}\left(\varphi_{+}(\tau)\right)+\gamma_{1} \varphi_{+}^{\frac{1}{2}}(\tau) \psi_{0}^{\prime}\left(\varphi_{+}(\tau)\right)+\cdots \quad \text { for } \tau \gg 1 .
$$

Since by the asymptotics (8.11) both terms are similar, this yields

$$
\frac{a_{0}^{\prime}}{a_{0}}=\hat{\gamma} \varphi_{+}^{\frac{3}{4}}(\tau) C_{4} \cos \left(d_{0} \varphi_{+}^{\frac{3}{2}}(\tau)+C_{5}\right)+\cdots \quad \text { for } \tau \gg 1 .
$$

We then obtain the following condition of irregularity of $(0,0)$ :

$$
(0,0) \text { is irregular if } \int^{\infty} \varphi_{+}^{\frac{3}{4}}(s) \cos \left(d_{0} \varphi_{+}^{\frac{3}{2}}(s)\right) \mathrm{d} s \text { converges. }
$$

There is no $\log -\log$ factor in the critical $R_{+}(t)$ and just a single log occurs: in view of the influence of the oscillatory factor $\cos (\cdot)$, the point

$(0,0)$ for $R_{+}(t)=(-t)^{\frac{1}{3}}|\ln (-t)|^{\gamma}:$ regular for $\gamma \leq \frac{4}{3}$, irregular for $\gamma>\frac{4}{3}$,

where, for the regular case, the corresponding oscillatory cut-off on $\varphi(\tau)$ according to the rule as in (7.31) is assumed, i.e., then $R_{+}(t)$ reads $\widetilde{R}_{+}(t)$.

Concerning the left-hand lateral boundary $\left\{x=-R_{-}(t), t \in[-1,0)\right\}$ (setting $\left.R_{+}(t) \equiv+\infty\right)$, the standard exponential decay behaviour of the kernel (8.11) as $y \rightarrow-\infty$ gives a result which is similar to the heat equation Gaussian (5.11), with $F(y) \sim \mathrm{e}^{-y^{2} / 4}$. Since the decay rate is slower in (8.11) governed by the power $|y|^{\frac{3}{2}}$, bearing in mind the integral criterion in (7.29), one can derive the following conclusion on the regularity:

$$
\int^{\infty} \ldots \mathrm{e}^{-d_{0}\left|\varphi_{-}(s)\right|^{3 / 2}} \mathrm{~d} s=\infty \Longrightarrow R_{-}(t)=\left(\frac{3 \sqrt{3}}{2}\right)^{\frac{2}{3}}(-t)^{\frac{1}{3}}[\ln |\ln (-t)|]^{\frac{2}{3}},
$$

where the constant $\left(\frac{3 \sqrt{3}}{2}\right)^{2 / 3}$ cannot be increased without losing the regularity of $(0,0)$. Since the kernel $F(y)$ is of constant sign in this limit, no oscillatory cut-off is necessary. Thus, the third-order PDE (8.5) is the last one, for which for a (one-sided) regularity criterion, there occurs a non-oscillatory kernel and the result looks similar to the heat equation one (3.9). Though no sub-solutions like 
(3.5) can be used, and, hypothetically, a majorizing order-preserving flow as in Appendix may be discussed. As we have seen, for the bi-harmonic equation (4.1), both lateral boundaries, in general, are assumed to undergo oscillatory cut-offs for the regularity. The same is true for the fifth-order linear dispersion equation and the tri-harmonic one

$$
u_{t}=u_{x x x x x} \quad \text { and } \quad u_{t}=u_{x x x x x x}, \text { etc.; }
$$

on their and others oscillatory properties, see [30, Chaps. 4 and 3].

More complicated spectral theory of multi-dimensional odd-order operators $\mathbf{B}^{*}$ and $\mathbf{B}$ is necessary for the study of the boundary regularity phenomena for the $N$-dimensional counterparts of linear dispersion PDEs such as

$$
u_{t}=(\Delta u)_{x_{1}}+\cdots \text { or } u_{t}=-\left(\Delta^{2} u\right)_{x_{1}}+\cdots \quad \text { in } Q_{0} .
$$

\subsection{On a quasilinear fourth-order diffusion equation}

Here we briefly explain necessary changes towards the regularity analysis for quasilinear diffusion-like PDEs. We take the 1D cubic 4th-order porous medium equation (the PME-4), as a basic model,

$$
u_{t}=-\left(u^{3}\right)_{x x x x} \text { in } Q_{0},
$$

where $Q_{0}$ is the same as in (4.1) and the Dirichlet boundary conditions now read

$$
u=\left(u^{3}\right)_{x}=0 \quad \text { at } x= \pm R(t), \quad-1 \leq t<0 .
$$

We specially have fixed a fully divergent diffusion model (8.27) with the monotone operator $-\left(u^{3}\right)_{x x x x}$ in the metric of $H^{-2}$, for which existence and uniqueness are guaranteed by classic theory; see Lions [55, Chap. 1,2]. Using the scaling (4.11) yields

$$
v_{\tau}=\mathbf{B}^{*}(v) \equiv-\left(v^{3}\right)_{\text {yyy }}-\frac{1}{4} y v_{y},
$$

where $\mathbf{B}^{*}(v)$ is now nonlinear. After the BL-analysis, we return to (8.29) to see how the linear operator $\mathbf{B}^{*}$ as in (4.12) will be recovered.

Boundary layer. The approach is similar, with the BL variables as in (7.2), where

$$
\xi=\frac{\varphi^{4 / 3}(\tau)}{\rho^{2 / 3}(\tau)}(1-z), \quad w(z, \tau)=\rho(\tau) g(\xi, \tau) \quad\left(z=\frac{y}{\varphi(\tau)}\right) .
$$

The BL rescaled equation such as (7.3) is easily recovered with the nonlinear operator

$$
g_{0}: \mathbf{A}(g) \equiv-\left(g^{3}\right)^{(4)}+\frac{1}{4} g^{\prime}=0, \quad \xi>0 ; \quad g=\left(g^{3}\right)^{\prime}=0 \text { at } \xi=0, \quad g(+\infty)=1 .
$$

The proof of existence of a positive solution $g(\xi)>0$ for $\xi>0$ of the problem (8.31) is not easy, but indeed doable by a standard shooting approach. To prove uniqueness, we rewrite this problem in a semilinear form 


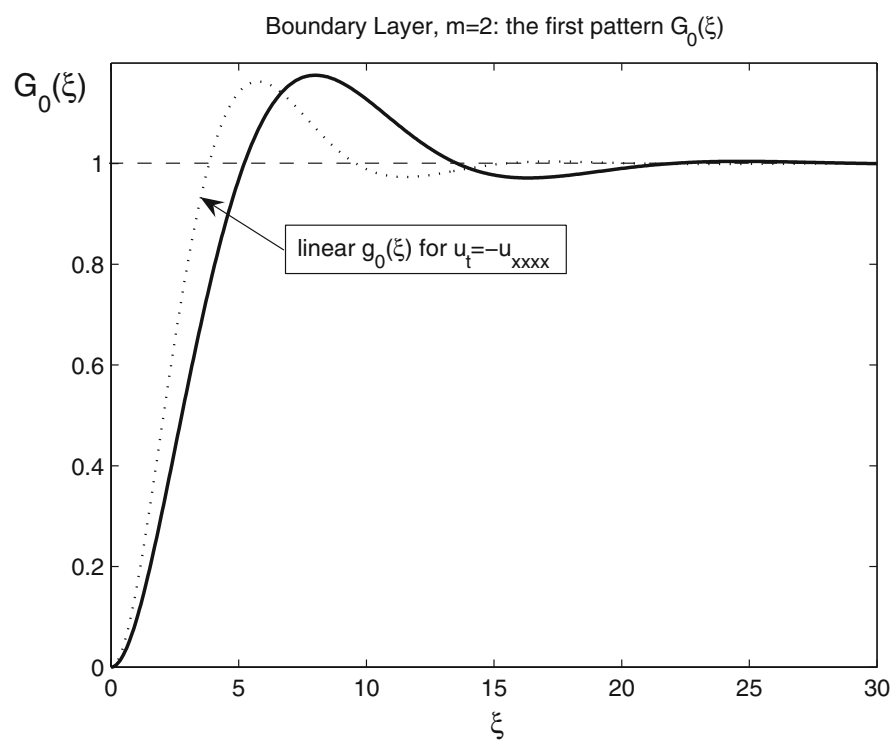

Figure 7 . The unique stationary solution $G_{0}(\xi)$ of the problem (8.32)

$$
G_{0}=g_{0}^{3}: \quad-G^{(4)}+\frac{1}{12}|G|^{-\frac{2}{3}} G^{\prime}=0, G=G^{\prime}=0 \text { at } \xi=0, \quad G(+\infty)=1 .
$$

On integration ones, this yields the equation with a monotone nonlinearity $G^{1 / 3}$ for $G>0$ :

$$
-G^{\prime \prime \prime}+\frac{1}{4} G^{\frac{1}{3}}=0 \quad(G>0) .
$$

Therefore, assuming existence of a second solution $\bar{G}>0$, we subtract the two equations and multiply by the difference $G-\bar{G}$ in $L^{2}$. Integrating by parts with using the boundary conditions in (8.32) and noting that the dispersion operator disappears yields

$$
\int\left(G^{\frac{1}{3}}-\bar{G}^{\frac{1}{3}}\right)(G-\bar{G})=0 \Longrightarrow G=\bar{G} .
$$

Proposition 8.1. The stationary BL-problem (8.31) has a unique solution $g_{0}(\xi)>0$ for $\xi>0$.

We next confirm these crucial properties of existence-uniqueness of $g_{0}(\xi)$ by using the solver bvp4c of the MatLab with the enhanced accuracy and tolerances (6.9). The sufficient for us reliable numerical justification is shown in Fig. 7, where the semilinear problem (8.32) is solved. The dotted line shows the "linear" $g_{0}(\xi)$ from Fig. 5, so that both linear and nonlinear profiles are similar, though correspond to different ODE mathematics. 
In general, stabilization in the full model, which is a quasilinear extension of (7.3), is rather technical and involved, but a class of such generic solutions forming the necessary boundary layer is indeed obtainable. Moreover, instead of the corresponding Proposition 7.1, which is difficult to prove in a sufficient generality, we may view this result as a definition of the class of generic solutions under consideration. For this class of solutions, the following holds on appropriate compact subsets near the boundary (cf. (7.22)):

$$
v(y, \tau)=\rho(\tau) g_{0}\left(\frac{\varphi^{4 / 3}(\tau)}{\rho^{2 / 3}(\tau)}\left(1-\frac{y}{\varphi(\tau)}\right)\right)+\cdots \quad \text { as } \tau \rightarrow+\infty .
$$

Thus, in the nonlinear case, the BL analysis is rather similar and demands only the extra scaling factor in the BL-variable $\xi$ in (8.30); cf. the linear one (7.2). Inner Region. This is also similar but there is a single nonlinear aspect that we have to address to. Thus, the only principal difference between (8.29) and (4.12) is that $\mathbf{B}^{*}(v)$ is a nonlinear operator, so we cannot immediately use the eigenfunctions expansion (7.17).

To this end, we recall that the BL-behaviour (8.34) actually implies that, in this critical case, we look for the so-called "mesa-like" asymptotics of solutions, which are supposed to be almost flat and to be independent of $y$ for $\tau \gg 1$ on compact subsets bounded away from the BL at $|y| \sim \varphi(\tau)$ (where (8.34) takes place). Such mesa-like asymptotics correspond to truly critical cases and are well-known in reaction-diffusion theory; see examples in [31, p. 59, 184]. According to this, it is correct to use the following approximation in the nonlinear term in $\mathbf{B}^{*}(v)$ :

$$
v^{3}=\rho^{2}(\tau) v(1+o(1)) \text { for } \tau \gg 1
$$

on compact subsets bounded away from thin domains, where the "non-flat" BL-expansion (8.34) is in charge. Then, via (8.35),

$$
-\left(v^{3}\right)_{\text {yyyy }} \mapsto-\rho^{2}(\tau) v_{\text {yyy }}+\cdots,
$$

so that, to get back to the linear operator $\mathbf{B}^{*}$ in the equation, one needs to rescale the spatial variable as follows:

$$
v(y, \tau)=w(\eta, \tau), \quad \text { where } \eta=\frac{y}{\sqrt{\rho(\tau)}}<\hat{\varphi}(\tau)=\frac{\varphi(\tau)}{\sqrt{\rho(\tau)}} \rightarrow+\infty .
$$

Therefore, $w(\eta, \tau)$ solves the equation that in leading terms coincides with (7.16),

$$
w_{\tau}=\mathbf{B}^{*} w+\frac{\rho^{\prime}}{\rho} \frac{1}{2} \eta w_{\eta}+\cdots
$$

where $+\cdots$ denotes higher-order terms due to the sharp approximation (8.35). In comparison with the linear case (7.16), we observe the additional second term of the order $O\left(\frac{\rho^{\prime}}{\rho}\right)$ for $\tau \gg 1$, which is clearly negligible in view of (4.7)-(4.10). Moreover, $w_{\eta} \equiv 0$ on the subspace spanned by $\psi_{0}^{*}=1$, for which (8.35) and (7.5) hold. 
We next introduce $\hat{w}$ according to the cut-off as in (7.14) at $\eta=\hat{\varphi}(\tau) \rightarrow+\infty$ given in (8.36). Then performing it first for the quasilinear equation for $w$ and next using the linearization as in (8.37), we arrive at

$$
\begin{aligned}
\hat{w}_{\tau}= & \mathbf{B}^{*} \hat{w}+\frac{\rho^{\prime}}{\rho} \frac{1}{2} \eta \hat{w}_{\eta} \\
& +\frac{1}{\rho^{2}}\left[\left.\left(w^{3}\right)_{\eta \eta \eta}\right|_{\eta=\hat{\varphi}} \delta(\eta-\hat{\varphi})+\left.\left(w^{3}\right)_{\eta \eta}\right|_{\eta=\hat{\varphi}} \delta^{\prime}(\eta-\hat{\varphi})\right]+\ldots
\end{aligned}
$$

Using in the last terms the BL estimates following from (8.34) (similar to (7.24)), and, as usual, applying (7.23) for the class of generic solutions, the eigenfunction expansion (7.17) for (8.38) yields the following asymptotic ODE for the first Fourier coefficient $\left(a_{0}>0\right)$ :

$$
a_{0}^{\prime}(\tau)=\gamma_{2} \sqrt{a_{0}(\tau)} \varphi(\tau) \psi_{0}\left(\frac{\varphi(\tau)}{\sqrt{a_{0}}}\right)+\gamma_{1} a_{0}^{\frac{2}{3}}(\tau) \varphi^{\frac{2}{3}}(\tau) \psi_{0}^{\prime}\left(\frac{\varphi(\tau)}{\sqrt{a_{0}}}\right)+\cdots .
$$

It follow that, unlike a simpler equation (7.25), due to the extra "nonlinear" scaling (8.36), the Fourier coefficient $a_{0}(\tau)$ in (8.39) is now entering the oscillatory rescaled kernel $\psi_{0}$ and its derivative $\psi_{0}^{\prime}$. Since $\varphi(\tau) \rightarrow+\infty$ and $a_{0}(\tau) \rightarrow 0$ for the regularity (see (7.26)), the first term in (8.39) is leading that yields on substitution the asymptotics (5.13), (5.14):

$$
a_{0}^{\prime}=\hat{\gamma} a_{0}^{\frac{2}{3}} \varphi^{\frac{2}{3}} \mathrm{e}^{-d_{0}\left(\varphi / \sqrt{a_{0}}\right)^{4 / 3}} C_{3} \cos \left[b_{0}\left(\frac{\varphi}{\sqrt{a_{0}}}\right)^{\frac{4}{3}}+C_{4}\right]+\cdots .
$$

The asymptotic analysis of this ODE is similar. For instance, the following rate of convergence is obtained for the unperturbed "Gaussian" parabola:

$$
\varphi(\tau) \equiv 1: \quad a_{0}(\tau) \sim 3^{\frac{3}{2}} 2^{-\frac{11}{2}}(\ln \tau)^{-\frac{3}{2}} \rightarrow 0,
$$

which shows the obvious result that the fundamental kernel parabolic shape for the shrinking point of $Q_{0}$ is regular.

In general, taking into account the main exponential term in (8.40) only, i.e., fixing the simplest approximating model

$$
a_{0}^{\prime}=-\mathrm{e}^{-d_{0}\left(\varphi / \sqrt{a_{0}}\right)^{4 / 3}} \quad\left(a_{0}(\tau)>0\right),
$$

we see that the critical $R(t)$ corresponds to

$$
\tilde{R}_{*}(t) \sim C(-t)^{\frac{1}{4}}[\ln |\ln (-t)|]^{\frac{3}{4}} \quad \text { as } t \rightarrow 0^{-},
$$

where $C>0$ is arbitrary. Of course, unlike the linear case in (7.33), the constant $C$ in (8.43) cannot play a role in view of the scaling invariance of the PDE (8.27),

$$
u=A \hat{u}, \quad x=\sqrt{A} \hat{x} \quad(A>0),
$$

which changes the constant $C$ without changing the regularity of the point. Similar to (3.18)-(3.20), the scaling (8.44) itself implies (8.43) by choosing $A(t)=a_{0}(\tau)$ from (8.41). 
It is not difficult to perform a more detailed study of the original ODE (8.40), though a too thorough analysis seems excessive. As usual, for the regularity, an oscillatory cut-off is necessary. Thus, it turns out that, for the quasilinear (8.27) and linear (4.1) fourth-order parabolic equations, the regularity conditions (including the concepts and the methods) of boundary regularity analysis can be obtained in similar lines. (TFE-4)

An analogous study can be done for the non-divergent thin film equation

$$
u_{t}=-\left(u^{2} u_{x x x}\right)_{x}
$$

though its existence-uniqueness theory is less developed, so the results will be more formal. The same techniques apply to the TFE- $(2,2)$, with other distributions of the inner and outer derivatives in the differential form

$$
u_{t}=-\left(u^{2} u_{x x}\right)_{x x},
$$

and to other PDEs with more general nonlinearities. Extensions to various quasilinear counterparts of the $2 m$ th-order parabolic equations (1.4) are also possible on the basis of the non self-adjoint spectral theory in [15].

\subsection{Fourth-order hyperbolic equation: regularity via Hermitian spectral theory for a pencil}

We recall that operator pencil theory fully occurred already in the regularity study for elliptic PDEs [44], and was later developed and extended in many papers; see $[24,45,47,48,65]$ for references. Boundary regularity for hyperbolic PDEs is definitely less developed; see last pages of Kondrat'ev-Oleinik's survey [45] of 1983 for some references. Note that, for fixed standard characteristic paraboloids posed for (8.45), the regularity problem falls into the scope of the results of Kondrat'ev's "parabolic" paper [43]. It seems that the case of "expanding paraboloids" as $t \rightarrow 0^{-}$, similar to the above cases for the bi-harmonic equations and others, was not treated before by the same reasons: the lack of proper spectral theory and matching via a boundary layer approach, which are very difficult to fully justify.

Here, according to the principle (1.8), we very briefly discuss a new type of operator pencils that is needed to tackle other regularity problems. The simplest such model satisfying (1.8) is the linear fourth-order hyperbolic (wave) equation

$$
u_{t t}=-u_{x x x x} \quad \text { in } Q_{0}
$$

also known as the 1D linear beam equation. We consider the same Dirichlet problem as in (4.1) with two bounded initial functions $u(x,-1)=u_{0}(x)$ and $u_{t}(x,-1)=u_{1}(x)$. Concerning the fundamental solution and necessary spectral properties, we follow [26].

Thus, the fundamental solution of (8.45) has the self-similar form

$$
b_{0}(x, t)=\sqrt{t} F(y), \quad y=\frac{x}{\sqrt{t}}, \quad \text { where } b_{0}(x, 0)=0, \quad b_{0 t}(x, 0)=\delta(x)
$$


in the sense of bounded measures. The rescaled kernel $F_{0}=F_{0}(|y|)$ is symmetric and solves the ODE

$$
\mathbf{B} F \equiv-F^{(4)}-\frac{1}{4} F^{\prime \prime} y^{2}-\frac{1}{4} F^{\prime} y+\frac{1}{4} F=0 \quad \text { in } \mathbb{R}, \quad \int F=1 .
$$

Integrating (8.47) once yields

$$
-F^{\prime \prime \prime}-\frac{1}{4} F^{\prime} y^{2}+\frac{1}{4} F y=0 \Longrightarrow F(y)=\frac{1}{2 \pi} \int_{0}^{\infty} \frac{\sin z \cos (\sqrt{z} y)}{z^{3 / 2}} \mathrm{~d} z .
$$

A WKBJ-type asymptotic analysis of the ODE (8.48) yields the behaviour like (1.8):

$$
F(y) \sim C_{1} y^{-\frac{11}{13}} \cos \left(\frac{y^{2}}{4}+C_{2}\right) \quad \text { as } y \rightarrow+\infty, \quad C_{1} \neq 0 .
$$

Similar to (4.11), the first scaling is

$$
\begin{gathered}
u(x, t)=v(y, \tau), \quad y=\frac{x}{\sqrt{-t}}, \quad \tau=-\ln (-t), \\
v_{\tau \tau}+v_{\tau}+v_{\tau y} y=\mathbf{B}^{*} v \equiv-v_{y y y y}-\frac{1}{4} y^{2} v_{y y}-\frac{3}{4} y v_{y} .
\end{gathered}
$$

Not paying enough attention to the functional setting of the operators involved in weighted $L^{2}$-spaces [26], we concentrate on the polynomial eigenfunctions of a quadratic pencil to appear; see Markus [58] for necessary concepts and theory of linear operator pencils. Namely, to find eigenfunctions, we set in (8.50)

$$
w(y, \tau)=\mathrm{e}^{\lambda_{k} \tau} \psi_{k}^{*}(y) \Longrightarrow \mathbf{C}^{*}\left(\lambda_{k}\right) \psi_{k}^{*} \equiv \mathbf{B}^{*} \psi_{k}^{*}-\left(\lambda_{k}^{2}+\lambda_{k}\right) \psi_{k}^{*}-\lambda_{k}\left(\psi_{k}^{*}\right)^{\prime} y=0
$$

Looking for finite polynomial eigenfunctions (recall that one needs two sets of eigenfunctions $\Psi^{*}=\left\{\psi_{k}^{*}(y), \phi_{k}^{*}(y)\right\}$ as well as the adjoint ones $\Psi=\left\{\psi_{k}(y), \phi_{k}(y)\right\}$ $[26])$,

$$
\psi_{k}^{*}(y), \phi_{k}^{*}(y)=y^{k}+\cdots \text { are } k \text { th-order polynomials, }
$$

substituting into (8.51), and keeping the higher-degree terms $\sim y^{k}$ yields the following quadratic equation for eigenvalues:

$$
\lambda_{k}^{2}+(k+1) \lambda_{k}+\frac{k(k-1)}{4}+\frac{3 k}{4}=0
$$

This gives two, shifted by -1 , series of eigenvalues,

$$
\lambda_{k}^{+}=-\frac{k}{2} \quad \text { and } \quad \lambda_{k}^{-}=-\frac{k}{2}-1 \quad \text { for } k=0,1,2, \ldots
$$


Let us specify a half of those generalized Hermite polynomials, which is a complete closed set. There is another one obtained in a similar manner, which is obviously necessary for the second-order in $t$ PDE (8.45).

Proposition 8.2. The eigenfunctions of the pencil (8.51) with the spectrum $\sigma_{+}=$ $\left\{\lambda_{k}^{+}\right\}$in (8.54) are given by the normalized polynomials

$$
\psi_{k}^{*}(y)=\frac{1}{\sqrt{k !}}\left[y^{k}+\sum_{j=1}^{\left[\frac{k}{4}\right]} \frac{1}{3^{j} j !}\left(y^{k}\right)^{(4 j)}\right], \quad k=0,1,2, \ldots
$$

Assuming as usual that $\varphi(\tau)$ is a slow growing function (so (4.7), (4.8),... hold), we introduce the variable $z$ in (7.1) and the resulting BL-variables as in (7.2), where

$$
\xi=\varphi^{2}(\tau)(1-z), \quad z=\frac{y}{\varphi(\tau)}, \quad D_{y}=-\varphi(\tau) D_{\xi}
$$

We suppose that the class of generic solutions admitting a BL-representation is under scrutiny and perform the cut-off as in (7.14), where $\hat{v}_{\tau \tau}=v_{\tau \tau} H$, by the expansion (7.20). We currently take into account the first coefficient only, with $\langle 1, F\rangle=1$ by the orthonormality, over the first half (8.55) of all the polynomials, and this defines the class of solutions involved. Then, eventually, by the asymptotics (8.49), we get

$$
a_{0}^{\prime \prime}+a_{0}^{\prime}+\cdots \sim \gamma_{1} C_{3} a_{0} \varphi^{\frac{28}{13}} \cos \left(\frac{\varphi^{2}}{4}+C_{4}\right)+\cdots \quad\left(\frac{28}{13}=3-\frac{11}{13}\right),
$$

where we omit all smaller terms containing $\varphi^{\prime}, \varphi^{\prime \prime}$, and others. For slow varying coefficients $a_{0}(\tau)$, the leading term is indeed $a_{0}^{\prime}$, so, in this critical case, the governing ODE is

$$
\frac{a_{0}^{\prime}}{a_{0}} \sim \hat{\gamma} C_{3} \varphi^{\frac{28}{13}} \cos \left(\frac{\varphi^{2}}{4}+C_{4}\right)+\cdots \Longrightarrow \ln \left|a_{0}(\tau)\right| \sim \int^{\tau} \varphi^{\frac{28}{13}} \cos \left(\frac{\varphi^{2}}{4}+C_{4}\right) \mathrm{d} \tau .
$$

Clearly, unlike (8.21), by setting $\varphi^{2}(\tau)=s$, we see from (4.7) that a converging integral for the irregularity is impossible. However, this does not mean a "total" regularity. Actually, this again implies that the regularity issue here is rather subtle and, as usual for oscillatory kernels, can be achieved by a proper oscillatory cut-off of suitable slow growing $\varphi(\tau)$. For general $\varphi(\tau)$, a more delicate analysis of the ODE (8.57) with all the terms included is necessary, which leads to some technical questions. A full justification of the general approach then becomes difficult and even questionable.

\section{Acknowledgments}

The author would like to thank M. Kelbert for a fruitful discussion of the regularity-probability PDE issues and interesting historical comments on Khinchin's 
results in 1924 and 1936 [40]. The author also thanks V.G. Maz'ya for help concerning general concepts of boundary regularity theory and for pointing out Feigin's earlier results [22], S.R. Svirshchevskii for getting early and rare V.P. Mihailov's papers (in Russian) in 1961-1963, and finally to A. Kyprianou for a short but informative conversation on Petrovskii's and Khinchin's contributions of the 1920s and 30s to the LIL and other related PDE-probability issues.

\section{Appendix: Comment: on using majorizing order-preserving operators}

It is clear that the main difficulty in establishing optimal regularity/irregularity criteria for higher-order linear parabolic PDEs such as (4.1), (1.4) (or third-order dispersion ones in (8.5) and (8.25) to be studied later on) is the fact that these flows do not obey the Maximum Principle and do not exhibit order-preserving features. As is well-known, the latter ones have been key for the heat equation (3.1) and other nonlinear second-order diffusion-reaction-absorption-convection... equations; see references in $[1,2,34,70]$.

Therefore, in parallel with going into eigenfunction expansion techniques for solving the regularity problem for (4.1), we cannot avoid a temptation to briefly discussing a rather formal opportunity to deal with the traditional techniques based on the Maximum Principle even for the higher-order parabolic flow. This is about the idea of majorizing order-preserving flows for higher-order parabolic equations [29], which we explain relative to the poly-harmonic flow (1.4). Namely, given the rescaled oscillatory kernel (5.5), we construct the corresponding majorizing kernel $\bar{F}(y)>0$ such that

$$
|F(y)| \leq D_{m} \bar{F}(y) \quad \text { in } \mathbb{R}^{N}, \quad \text { where } \int \bar{F}=1,
$$

and $D_{m}>1$ is a constant called the order deficiency (or order defect) of this majorizing kernel. It is clear that the optimal order deficiency is $\left(D_{1}^{*}=1\right.$ since $F>0$ in $(5.11))$

$D_{m}^{*}=\int|F|>1, \quad$ corresponding to the non-smooth kernel $\quad \bar{F}(y)=\frac{1}{D_{m}^{*}}|F(y)|$.

Sometimes, dealing with not $C^{\infty}$ kernels $\bar{F}(y)$ is not convenient, especially when determining its eigenfunctions by differentiation via (5.17), with $F \mapsto \bar{F}$, etc.

We then introduce the corresponding majorizing order-preserving integral evolution equation in $\mathbb{R}^{N} \times \mathbb{R}_{+}$(it does not have a PDE representation in general) defined by the formal convolution

$$
\bar{u}(t)=\bar{b}(t) * \bar{u}_{0}, \text { with } \bar{u}_{0}(x) \geq 0, \quad \text { where } \bar{b}(x, t)=t^{-\frac{1}{2 m}} \bar{F}(y), \quad y=\frac{x}{t^{1 / 2 m}} .
$$


The corresponding comparison theorem is as follows [29]:

$$
\left|u_{0}(x)\right| \leq \frac{1}{D_{m}} \bar{u}_{0}(x) \quad \text { in } \mathbb{R}^{N} \Longrightarrow|u(x, t)| \leq \bar{u}(x, t) \quad \text { in } \mathbb{R}^{N} \times \mathbb{R}_{+} .
$$

The proof is straightforward in view of (A.1) via comparison of the solution of (A.3) with the parabolic flow

$$
u(x, t)=b(x-\cdot, t) * u_{0}(\cdot) \equiv t^{-\frac{1}{2 m}} \int F\left(\frac{x-y}{t^{1 / 2 m}}\right) u_{0}(y) \mathrm{d} y \quad \text { in } \mathbb{R}^{N} \times \mathbb{R}_{+} .
$$

Indeed, subtracting (A.3) and (A.5) yields for the difference the desired inequality:

$$
\begin{aligned}
w(x, t) & =\bar{u}(x, t)-u(x, t)=t^{-\frac{1}{2 m}} \int\left[\bar{F}(\cdot) \bar{u}_{0}-F(\cdot) u_{0}\right] \\
& \geq t^{-\frac{1}{2 m}} \int\left[\bar{F}(\cdot) \bar{u}_{0}-\frac{1}{D_{m}}|F(\cdot)| \bar{u}_{0}\right]=t^{-\frac{1}{2 m}} \int\left[\frac{D_{m} \bar{F}(\cdot)-|F(\cdot)|}{D_{m}} \bar{u}_{0}\right] \geq 0,
\end{aligned}
$$

by the definition of the majorizing kernel (A.1). Since $\bar{F}>0$, i.e., the integral majorizing evolution (A.3) is order-preserving, solutions $\bar{u}(x, t)$ admit constructing super-solutions $\bar{U}(x, t)$ in the standard sense, i.e., the following comparison holds:

$$
\bar{U}(t) \geq \bar{b}(t) * \bar{u}_{0} \Longrightarrow 0<\bar{u}(x, t) \leq \bar{U}(x, t) \quad \text { in } \mathbb{R}^{N} \times \mathbb{R}_{+} .
$$

In [29], such comparison ideas resolved some asymptotic problems for semilinear parabolic equations related to Fujita critical exponents and around.

Obviously, to construct a majorizing comparison theory for the IBVPs in domains such as $Q_{0}$, further estimates of "Green's function" corresponding to the "majorizing non-translational flow" (A.3) in $Q_{0}$ (as usual, such an estimate is expected to be related to (A.1)) are necessary. These are difficult questions, which are not discussed here. We just mention that such a true possibility would allow, in a reasonable simpler (and rigorous!) way, to get a proper upper estimates on solutions in $Q_{0}$ to prove that $u(0,0)=0$, which would guarantee its regularity, by constructing proper super-solutions. As a formal clue, it would be very efficiently and attractively easy to use Petrovskii's ansatz (3.5),

$$
\bar{U}(x, t) \sim-\frac{1}{|\ln (-t)|^{1+\varepsilon_{1}}} \mathrm{e}^{-d_{0}|x|^{\frac{2 m}{2 m-1}} /(-t)^{\frac{1}{2 m-1}}}+\frac{1}{\ln |\ln (-t)|},
$$

where we have replaced the Gaussian structure in (3.5) by that in the estimate (5.7), which is supposed to be true for the majorizing positive kernel $\bar{F}(y)$. As we have shown (cf. (7.33) for $m=2$ ), the space-time structure (A.8) would give a correct critical behaviour for $R(t)$ as $t \rightarrow 0^{-}$. Unfortunately, we do not know if this can be justified in such a comparison way (an open problem, and a rather attractive one). We must admit that, besides some other fundamental open questions, in any case, unlike (3.5), using (A.8) for any $m \geq 2$ assumes complicated integral calculus to establish the validity of the integral inequality in (A.7). More 
importantly, such an integral majorizing approach cannot be used for any lower bound on $u(x, t)$ to get the irregularity counterpart, when $|u(0,0)|>0$. As one can see, corresponding alternative "minorizing" theory in similar lines cannot be constructed in principle. Therefore, we had no intention and in fact necessity to check whether functions such as (A.8) can satisfy (A.7) or similar integral inequalities. However, we state the following principal open problem: ${ }^{19}$ Is there any hope to get sharp regularity results by using majorizing-like calculus associated with some integral operator in $Q_{0}$ with a positive kernel?

\section{References}

[1] Abdulla, U.G.: Reaction-diffusion in irregular domains. J. Differ. Equ. 164, 321354 (2000)

[2] Abdulla, U.G.: Multidimensional Kolmogorov-Petrovsky test for the boundary regularity and irregularity of solutions to the heat equation. Bound. Value Probl. 2, 181199 (2005)

[3] Abdulla, U.G.: Wiener's criterion at $\infty$ for the heat equation. Adv. Differ. Equ. 13, 457-488 (2008)

[4] Aref'ev, V.N., Bagirov, L.A.: Solutions of the heat equation in domains with singularities. Math. Notes 64, 139-153 (1998)

[5] Arkhangelskii, A.V., Tikhomirov, V.M.: Pavel Samuilovich Urysohn (18981924). Russ. Math. Surv. 55, 875-892 (1998)

[6] Azizov, T.Y., Iokhvidov, I.S.: Linear Operators in Spaces with an Indefinite Metric. Wiley-Interscience, Chichester/Singapore (1989)

[7] Barbatis, G.: Explicit estimates on the fundamental solution of higher-order parabolic equations with measurable coefficients. J. Differ. Equ. 174, 442-463 (2001)

[8] Barbatis, G.: Sharp heat-kernel estimates for higher-order operators with singular coefficients. Proc. Edinb. Math. Soc. (2) 47, 53-67 (2004)

[9] Bidaut-Véron, M.-F., Galaktionov, V., Grillot, P., Véron, L.: Singularities for a 2-dimensional semilinear elliptic equation with a non-Lipschitz nonlinearity. J. Differ. Equ. 154, 318-338 (1999)

[10] Birman, M.S., Solomjak, M.Z.: Spectral Theory of Self-Adjoint Operators in Hilbert Space. D. Reidel, Dordrecht/Tokyo (1987)

\footnotetext{
${ }^{19}$ Personally, the author suspects that this is not possible, especially since the "cut-off" must be then inherited by the method (how and why?); though then proving the negative result (a principal non-applicability of such barrier techniques for $m \geq 2$ ) would be also important.
} 
[11] Borsuk, M.: Degenerate elliptic boundary-value problems of second order in nonsmooth domains. J. Math. Sci. 146, 6071-6212 (2007)

[12] Carleman, T.: Über das Neumann-Poincaresche Problem für ein Gebiet mit Ecken. Dissertation, Uppsala (1916)

[13] Courant, R., Hilbert, D.: Methods of Mathematical Physics, vol. II. In: Courant, R. (ed.) Partial Differential Equations. Interscience Publication, New York (1962)

[14] Durrett, R.: Probability: Theory and Examples. Wadworth \& Brooks/Cole Adv. Books \& Software, Pacific Grove, CA (1991)

[15] Egorov, Yu.V., Galaktionov, V.A., Kondratiev, V.A., Pohozaev, S.I.: Global solutions of higher-order semilinear parabolic equations in the supercritical range. Adv. Differ. Equ. 9, 1009-1038 (2004)

[16] Eidelman, S.D.: Parabolic Systems. North-Holland Publ. Comp., Amsterdam (1969)

[17] Eidelman, S.D.: Parabolic equations. In: Modern Problems of Math. Fundam. Achiev., vol. 63. (Part. Differ. Equat.). VINITI AN SSSR: 1990, Moscow, p. 244. English transl.: J. Soviet Math. (1991)

[18] Eilstein, S.: On weighted fractional integral inequalities. J. Funct. Anal. 185, 342$366(2001)$

[19] Ellias, U.: Eigenvalue problems for the equation $L y+\lambda p(x) y=0$. J. Differ. Equ. 29, 28-57 (1978)

[20] Evans, L., Gariepy, R.F.: Wiener's criterion for the heat equation. Arch. Ration. Mech. Anal. 78, 293-314 (1982)

[21] Faminskii, A.V.: On the mixed problem for quasilinear equations of the third order. J. Math. Sci. 110, 2476-2507 (2002)

[22] Fě̈gin, V.I.: Boundary value problems for quasi-elliptic equations in noncylindrical regions. Sov. Math. Dokl. 12, 634-638 (1971)

[23] Fernandes, R.S., Galaktionov, V.A.: Very singular similarity solutions and Hermitian spectral theory for semilinear odd-order PDEs. submitted

[24] Frehse, J., Kassmann, M.: Nonlinear partial differential equations of fourth order under mixed boundary conditions. Math. Z. 254, 33-54 (2006)

[25] Galaktionov, V.A.: Sturmian nodal set analysis for higher-order parabolic equations and applications. Adv. Differ. Equ. 12, 669-720 (2007)

[26] Galaktionov, V.A.: Hermitian spectral theory and blow-up patterns for a fourth-order semilinear Boussinesq equation. Stud. Appl. Math. 121, 395-431 (2008) 
[27] Galaktionov, V.A.: On regularity of a boundary point for higher-order parabolic equations: towards Petrovskii-type criterion by blow-up approach. Preprint, 2008 (arXiv:0901.3986)

[28] Galaktionov, V.A.: On $\sqrt{\log \log }$ blow-up in higher-order reaction-diffusion and wave equations: a formal "geometric" approach. Phys. D, to appear (arXiv:0901.4314)

[29] Galaktionov, V.A., Pohozaev, S.I.: Existence and blow-up for higher-order semilinear parabolic equations: majorizing order-preserving operators. Indiana University Math. J. 51, 1321-1338 (2002)

[30] Galaktionov, V.A., Svirshchevskii, S.R.: Exact Solutions and Invariant Subspaces of Nonlinear Partial Differential Equations in Mechanics and Physics. Chapman \& Hall, Boca Raton (2007)

[31] Galaktionov, V.A., Vazquez, J.L.: A Stability Technique for Evolution Partial Differential Equations. A Dynamical Systems Approach. Progress in Nonlinear Differential Equations and Their Applications, vol. 56, Birkhäuser, Boston/Berlin (2004)

[32] Gevrey, M.: Sue les équations aux dérivées partielles due type parabolique. J. Math. Pure Appl. Paris (6), 9, 305-471 (1913); 10, 105-148 (1914)

[33] Grigor'yan, A., Kelbert, M.: On Hardy-Littlewood inequality for Brownian motion on Riemannian manifolds. J. Lond. Math. Soc. (2) 62, 625-639 (2000)

[34] Herrero, M.A., Ughi, M., Velázquez, J.J.L.: Approaching a vertex in a shrinking domain under a nonlinear flow. Nonlinear Differ. Equ. Appl. 11, 1-28 (2004)

[35] Ivasishen, S.D.: Estimates of the Green function of the homogeneous first boundary value problem for a parabolic equation in a noncylindrical domain. Ukr. Mat. Zh. 21, 15-27 (1969)

[36] Kato, T.: Perturbation Theory for Linear Operators. Springer-Verlag, Berlin (1976)

[37] Keldysh, M.V.: Sur le problème de Dirichlet. Doklady Acad. Nauk SSSR (N.S.) 32, 308-309 (1941)

[38] Keldysh, M.V.: On the solvability and stability of the Dirichlet problem. Uspehi Matem. Nauk, 8, 171-231 (1941) (In Russian; MathRev. Reviever: Tamarkin, J.D.); Am. Math. Soc. Transl. (2), 51, 1-73 (1966)

[39] Kellogg, O.D.: Foundations of Potential Theory. Frederick Ungar Publication Co., New York (1929)

[40] Khinchin, A.Y.: Asymptotic Laws in Probability Theory. ONTI, Moscow, 1936. In $[74$, p. 57] and [75, p. 385], the following earlier Khinchin's work (a book) is mentioned: Khintchine, A., Asymptotische Gesetze der Wahrscheinlichkeitsrechnung, Ergebnisse, 1933, and Petrvskii improved his result on the irregularity for the curve $x^{2}=4(1+\varepsilon)|t| \ln |\ln | t||, \varepsilon>0$.

[41] Kolmogorov, A.N., Petrovskii, I.G., Piskunov, N.S.: Study of the diffusion equation with growth of the quantity of matter and its application to a biological problem. 
Byull. Moskov. Gos. Univ. Sect. A, 1, 1-26 (1937). English. transl. In: Pelcé, P. (ed.) Dynamics of Curved Fronts, pp. 105-130. Academic Press, Inc., New York (1988)

[42] Kondrat'ev, V.A.: On the solvability of the first boundary value problem for elliptic equations. Sov. Math. Dokl. 2, 127-130 (1961)

[43] Kondrat'ev, V.A.: Boundary value problems for parabolic equations in closed regions. Trans. Moscow Math. Soc., vol. 15, Am. Math. Soc., Providence, RI, 1966, pp. $450-504$

[44] Kondrat'ev, V.A.: Boundary value problems for elliptic equations in domains with conical or angular points. Trans. Moscow Math. Soc., vol. 16, Am. Math. Soc., Providence, RI, 1967, pp. 227-313

[45] Kondrat'ev, V.A., Olěinik, O.A.: Boundary-value problems for partial differential equations in nonsmooth domains. Russ. Math. Surv. 38, 1-86 (1983)

[46] Kozlov, V.A., Maz'ya, V.G.: Asymptotic formula for solutions to elliptic equations near the Lipschitz boundary. Ann. di Matem. 184, 185-213 (2005)

[47] Kozlov, V.A., Maz'ya, V.G., Rossmann, J.: Elliptic Boundary Value Problems in Domains with Point Singularities. Math. Surv. Monogr., vol. 52, Am. Math. Soc., Providence, RI (1997)

[48] Kozlov, V.A., Maz'ya, V.G., Rossmann, J.: Spectral Problems with Corner Singularities of Solutions to Elliptic Equations. Math. Surv. Monogr., vol. 85, Am. Math. Soc., Providence, RI (2001)

[49] Labutin, D.A.: Wiener regularity for large solutions of nonlinear equations. Ark. Mat. 41, 307-339 (2003)

[50] Landis, E.M.: Necessary and sufficient conditions for regularity of a boundary point in the Dirichlet problem for the heat conduction equation. Sov. Math. Dokl. 10, 380 384 (1969)

[51] Lebesgue, H.: Sur le problemè de Dirichlet. Rend. Circolo Mat. di Palermo 24, 371$402(1907)$

[52] Lebesgue, H.: Sur le problemè de Dirichlet. Compt. Rendus Acad. Sci. Paris 154, 335-337 (1912)

[53] Lebesgue, H.: Sur de cas d'impossibilité du problemè de Dirichlet. Comp. Rend. Soc. Math. France 17, 48-50 (1913)

[54] Lebesgue, H.: Conditions de regularité, conditions d'irregularité, conditions d'impossibilité dans le problemè de Dirichlet. Compt. Rendus Acad. Sci. Paris 178, 349354 (1924)

[55] Lions, J.L.: Quelques méthodes de résolution des problèmes aux limites non linéaires. Dunod, Gauthier-Villars, Paris (1969) 
[56] Mamedov, I.T.: Boundary properties of solutions to second-order parabolic equations in domains with special symmetry. Math. Notes 70, 347-362 (2001)

[57] Marcus, M., Véron, L.: Maximal solutions of the equation $\Delta u=u^{q}$ in arbitrary domains. Compt. Rend. Acad. Sci. Paris Ser. I 344, 299-304 (2007)

[58] Markus, A.S.: Introduction to the Spectral Theory of Polynomial Operator Pencils. Transl. Math. Mon., vol. 71, Am. Math. Soc., Providence, RI (1988)

[59] Maz'ya, V.G.: On the continuity at a boundary point of solutions of quasi-linear elliptic equations. Vestnik Leningr. Univ. Math. Mech. Actronom. 25, 42-55 (1970); English transl. Vestnik Leningr. Univ. Math. 3, 225-242 (1976)

[60] Maz'ya, V.: Behaviour of solutions to the Dirichlet problem for the biharmonic operator at a boundary point. Equadiff IV (Proc. Czechoslovak Conf. Differ. Equat. Appl., Prague, 1977), Lecture Notes Math., vol. 703, pp. 250-262. Springer, Berlin (1977)

[61] Maz'ya, V.: Unsolved problems connected with the Wiener criterion. The Legacy of Norbert Wiener: A Centennial Symposium (Cambridge, MA, 1994), Proc. Symp. Pure Math., vol. 60, pp. 199-208, Am. Math. Soc., RI (1997)

[62] Maz'ya, V.: On the Wiener type regularity of a boundary point for the plyharmonic operator. Appl. Anal. 71, 149-165 (1999)

[63] Maz'ya, V.: The Wiener test for higher order elliptic equations. Duke Math. J. 115, 479-512 (2002)

[64] Maz'ya, V.G., Nazarov, S.A.: The apex of a cone can be irregular in Wiener's sense for a fourth-order elliptic equation. Math. Notes 39, 14-16 (1986)

[65] Maz'ya, V.G., Plamenevskii, B.A.: On the maximum principle for the biharmonic equation in a domain with conical points. Izv. Vyssh. Uchebn. Zaved. Mat., No. 2, 52-59 (1981)

[66] Mihailov, V.P.: The Dirichlet problem and the first mixed problem for a parabolic equation. Dokl. Akad. Nauk SSSR 140, 303-306 (1961)

[67] Mihailov, V.P.: The Dirichlet problem for a parabolic equation. I. Mat. Sb. (N.S.) 61(103), 40-64 (1963)

[68] Mihaillov, V.P.: The Dirichlet problem for a parabolic equation. II. Mat. Sb. (N.S.) 62(104), 140-159 (1963)

[69] Mikhailov, V.P.: Existence of the boundary value of a polyharmonic functions. Sib. Math. J. 46, 902-912 (2005)

[70] Mossino, J., Ughi, M.: On the regularity at shrinking points for the porous media equation. Nonlinear Differ. Equ. Appl. 7, 1-21 (2000)

[71] Naimark, M.A.: Linear Differential Operators, Part 1. Frederick Ungar Publication Co., New York (1967) 
[72] Nikol'skii, S.M.: Boundary properties of functions defined on a region with angular points. I, II, III. Mat. Sb. N. S., 40(82), 303-318 (1956); 43(85), 127-144 (1957); 45(87), 181-194 (1958); English Transl.: Am. Math. Soc. Transl., Series 2, vol. 83, AMS, Providence, R.I. (1969)

[73] Petrovsky, I.G.: Über das Irrfahrtproblem. Math. Ann. 109, 425-444 (1934)

[74] Petrowsky, J.: Über die Lösungen der ersten Randwertaufgabe der Wärmeleitungsgleichung. Učenye Zapiski Moscovsk. Gosud. Univ., No. 2, 55-59 (1934), Moscow, USSR (in German, with Russian summary)

[75] Petrovsky, I.G.: Zur ersten Randwertaufgabe der Wärmeleitungsleichung. Compos. Math. 1, 383-419 (1935)

[76] Petrovskii, I.G.: Lectures on Partial Differential Equations, 3rd edn. Gos. Izdat. Fiz.-Mat. Lit., Moscow (1961)

[77] Poincaré, H.: Sur le problème de la distribution électrique. Compt. Rendus Acad. Sci. Paris 104, 44-46 (1887)

[78] Rabinovich, V., Schulze, B.-W., Tarhanov, N.: Boundary value problems in oscillatory cuspidal wedges. Rocky Mount. J. Math. 34, 1399-1471 (2004)

[79] Samarskii, A.A., Galaktionov, V.A., Kurdyumov, S.P., Mikhailov, A.P.: Blow-up in Quasilinear Parabolic Equations. Walter de Gruyter, Berlin (1995)

[80] Solonnikov, V.A.: On boundary value problems for linear parabolic systems of differential equations of the general form. Trudy Steklov Math. Inst., vol. 83, Nauka, Moscow-Leningrad (1965)

[81] Tamarkin, J.D.: Some General Problems of the Theory of Ordinary Differential Equations and Series Expansion of Arbitrary Functions. Tipografia Frolovoi, M.P., Petrograd, 1917 (in Russian)

[82] Uryson, P.S.: Zur ersten Randwertaufgabe der Potentialtheorie. Math. Z. 23, 155$158(1925)$

[83] Vainberg, M.A., Trenogin, V.A.: Theory of Branching of Solutions of Non-Linear Equations. Noordhoff International Publication, Leiden (1974)

[84] Véron, L.: Singularities of Solutions of Second Order Quasilinear Equations. Pitman Res. Notes Math. Ser. vol. 353. Longman, Harlow (1996)

[85] Véron, L.: Elliptic Equations Involving Measures. Stat. Part. Differ. Equ., vol. I, 593-712. Handbook Differ. Equat., North-Holland, Amsterdam (2004)

[86] Wiener, N.: The Dirichlet problem. J. Math. Phys. Mass. Inst. Tech., 3, 127-146 (1924); reprinted In: Wiener, N., Collected Works with Commentaries, vol. I, ed. Masani, P., Mathematicians of Our Time 10. MIT Press, Cambridge, pp. 394-413 (1976) 
[87] Wood, I.: Maximal $L^{p}$-regularity for the Laplacian on Lipschitz domains. Math. Z. 255, 855-875 (2007)

[88] Zaremba, S.: Sur le principe de Dirichlet. Acta Math. 34, 293-316 (1911)

V. A. Galaktionov

Department of Mathematical Sciences,

University of Bath,

Bath BA2 7AY, UK

e-mail: vag@maths.bath.ac.uk

Received: 31 October 2008

Accepted: 15 April 2009 